\title{
Thermodynamic properties and homogeneous nucleation rates for surface-melted physical clusters
}

\author{
Richard B. McClurg and Richard C. Flagan \\ Spalding Laboratory (210-41), Division of Chemistry and Chemical Engineering (CN 9141), \\ California Institute of Technology, Pasadena, California 91125 \\ William A. Goddard III ${ }^{\text {a) }}$ \\ Materials and Process Simulation Center, Beckman Institute (139-74), Division of Chemistry and Chemical \\ Engineering (CN 9141), California Institute of Technology, Pasadena, California 91125
}

(Received 12 September 1995; accepted 19 July 1996)

\begin{abstract}
We predict the free energy of van der Waals clusters $\left(F_{n}\right)$ in the surface-melted temperature regime. These free energies are used to predict the bulk chemical potential, surface tension, Tolman length, and vapor pressure of noble gas crystals. Together, these estimates allow us to make definitive tests of the capillarity approximation in classical homogeneous nucleation theory. We find that the capillarity approximation underestimates the nucleation rate by thirty orders of magnitude for argon. The best available experiments are consistent with our calculation of nucleation rate as a function of temperature and pressure. We suggest experimental conditions appropriate for determining quantitative nucleation rates which would be invaluable in guiding further development of the theory. To make the predictions of $F_{n}$, we develop the Shellwise Lattice Search (SLS) algorithm to identify isomer fragments and the Linear Group Contribution (LGC) method to estimate the energy of isomers composed of those fragments. Together, SLS/LGC approximates the distribution of isomers which contribute to the configurational partition function (for up to 147-atom clusters). Estimates of the remaining free energy contributions come from a previous paper in this series. (C) 1996 American Institute of Physics. [S0021-9606(96)01940-X]
\end{abstract}

\section{INTRODUCTION}

The structure and dynamics of neutral, physically bound clusters containing 10 to a few hundred particles are critical to understanding homogeneous nucleation. To date, the only direct experimental probe of homogeneous nucleation is the so-called nucleation theorem. ${ }^{1}$ It can be used to determine the size and composition of the critical nucleus, but does not provide information on cluster properties. Since experimental study of clusters in this size range has proved difficult, it is useful to develop theoretical methods.

Noble gas clusters with a broad size distribution can be readily produced using an adiabatic expansion jet. ${ }^{2}$ Electron diffraction experiments ${ }^{3-5}$ show that small argon clusters formed in a jet adopt 5-fold symmetry. This contrasts with the bulk (fcc) structure that is observed for clusters larger than $N_{f c c}(T)$. At $32 \mathrm{~K}$ this limit is $N_{f c c}(T) \approx 750$ atoms ${ }^{3}$ and at $10 \mathrm{~K}, 1500 \leqslant N_{f c c}(T) \leqslant 3500$ atoms. ${ }^{4,5}$ Calculations suggest that $N_{f c c}(0 \mathrm{~K})=10^{5}$ atoms. ${ }^{6}$ Experiments are somewhat restricted by the distribution of cluster sizes produced in jets. In addition, it is difficult to obtain size-selected neutral clusters since few experimental methods successfully separate neutral clusters beams. ${ }^{7}$ Mass spectrometry has sufficient resolution to separate cluster beams into its components, but ionization of the clusters leads to structural changes in the clusters being studied. ${ }^{8}$ Neutralization of size-selected ionic clusters produces a narrow size distribution of clusters, but the vibrational energy of the neutralized clusters exceeds that of thermalized clusters at the temperature of the incoming

a) Author to whom correspondence should be addressed. cluster beam. ${ }^{9}$ Deflection of a neutral cluster beam with an atomic beam has been used to separate a cluster beam with atomic resolution for small clusters (less than 10 rare gas atoms ${ }^{10}$ or up to 13 molecules ${ }^{11}$ ). Many applications require knowledge of the properties of larger clusters. The deficiencies in the available experimental techniques necessitate theoretical approaches to study the energetics of neutral thermalized clusters.

Most cluster modeling and simulation studies use pairwise additive potentials to simplify the analysis. Even so, the $3 n$ degrees of freedom for a cluster of $n$ particles makes determining even the optimum structure challenging. Reviews by Hoare ${ }^{12}$ and Farges et al. ${ }^{13}$ summarize much of the work in finding candidates for minimum energy structures. More recently, structures have been proposed for each cluster size from 13 to 147 particles, ${ }^{14}$ and selected clusters with as many as several thousand particles have been studied. ${ }^{6,15}$ Since none of these studies involved exhaustive searches for the global minimum energy, each provides only an upper bound on the minimum potential energy. However, the consensus is that the low energy structures are well understood, making it reasonable to consider the dynamics of clusters using the $(3 n)$-dimensional surfaces based on these potentials.

Cluster thermodynamics have been studied using molecular dynamics (MD) $)^{3,12,16-19}$ and Monte Carlo $(\mathrm{MC})^{12,19-24}$ simulations, and statistical-mechanical modeling. ${ }^{12,18}$ All three approaches face a formidable challenge: sampling of the potential surface in sufficient detail to faithfully represent the thermodynamically accessible regions. Most approaches waste most of the effort on sampling 
unimportant regions. The number of local minima for a cluster of a given number of particles $(n)$ indicates the difficulty of this task. Tsai and Jordan identified 1328 minima for the 13-particle cluster, ${ }^{25}$ and Berry has suggested that the number of geometrically distinct minima grows exponentially with $n \cdot{ }^{26}$ With far too many minima for exhaustive sampling, simulations can become trapped in local potential wells. In this paper, we present an approach for simplifying this endeavor using insights gained from smaller systems to guide the modeling of larger systems.

In Sec. II we review pertinent insights (from simulations) concerning the structure of atomic clusters. In Sec. III we lay out the statistical-mechanical basis for the Shellwise Lattice Search (SLS) algorithm described in Secs. IV and V. In Sec. VI we present the linear group contribution (LGC) method used to estimate shell energies. Finally, we apply the results of SLS/LGC to the analysis of homogeneous nucleation experiments in Sec. VII.

\section{STRUCTURES}

The lowest energy minima of small atomic clusters have icosahedral structures. These structures were first studied by Mackay who characterized them as "a dense noncrystallographic packing of equal spheres.",27 The Mackay icosahedral structures are constructed by surrounding a central atom with successively larger icosahedral shells having the same orientation of their vertices. Shell number one has twelve atoms at the vertices of the icosahedron. The second shell contains twelve atoms at these vertices plus atoms on each of the 30 edges of the icosahedron for a total of 42 atoms in the second shell (a grand total of 55 atoms). Higherorder shells have additional atoms along the edges and in the faces of the icosahedral shell. In general, the number of atoms $n$ in a Mackay structure with $N$ shells is

$$
n=1+\sum_{x=1}^{N}\left(10 x^{2}+2\right)=\frac{1}{3}\left(10 N^{3}+15 N^{2}+11 N+3\right) .
$$

Thus, the first 8 Mackay icosahedral structures contain 13, 55, 147, 309, 561, 923, 1415, and 2057 atoms. These are called Magic Numbered Clusters. ${ }^{28}$ For clusters intermediate in size between the magic numbers, the excess particles reside on the surface of a dense icosahedral cluster.

Dense icosahedral clusters are solidlike at low temperatures because the atoms vibrate about a single minimum on the potential surface. Of course, these structures are not crystalline solids since they lack translational invariance. Honeycutt and Andersen ${ }^{16}$ and Berry et al. ${ }^{17-19}$ showed that slightly above the minimum energy, there are minima having icosahedral cores with one or more atoms "promoted" onto the surface. Such clusters with incomplete outer layers are called surface melted clusters because the surface atoms diffuse much more rapidly than core atoms. ${ }^{18}$ At still higher energies, the cluster samples liquidlike amorphous structures.

We have adopted the concept of inherent structures as proposed by Stillinger and Weber. ${ }^{29}$ In this approach, the (3n)-dimensional potential surface is divided conceptually

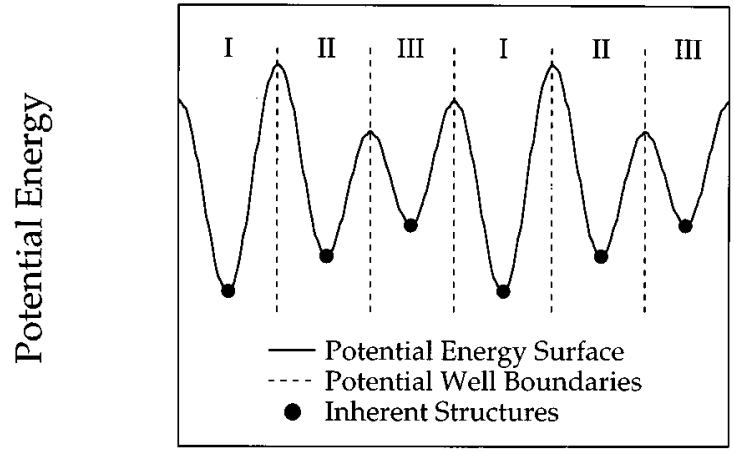

\section{Generalized Coordinate}

FIG. 1. Schematic potential surface illustrating the definitions of inherent structures (also called isomers) and potential wells around each isomer. For a cluster of $n$ particles, the $3 n$-dimensional potential surface can similarly be divided into potential wells, each with an inherent structure of a different structure. Structures that differ only by a permutation of the atoms are indistinguishable and therefore belong to the same isomer.

into regions corresponding to the potential wells of each of the geometrically distinguishable local minima on the potential surface. Geometrically distinguishable configurations cannot be made coincident by a combination of translation and/or rotation of the cluster and permutation of the atoms in the cluster. The structure at the bottom of the well is called the inherent structure for the well. Other configurations within a well are considered as vibrationally excited states of that well's inherent structure. For brevity we refer to the inherent structures as isomers. See Fig. 1 for an illustration of these definitions. This framework allows us to express the equilibrium thermodynamic functions for clusters as summations over isomers.

\section{CONFIGURATIONAL PARTITION FUNCTION}

The molar free energy for an ideal polyatomic gas,

$$
F=-R T \ln \left(\frac{q}{N_{a}}\right)
$$

is calculated ${ }^{30}$ from the single-cluster partition function, $(q)$ which can be separated into terms for translation $\left(q_{\mathrm{tr}}\right)$, rotation $\left(q_{\text {rot }}\right)$, vibration $\left(q_{\text {vib }}\right)$, degeneracy $\left(d_{j}\right)$, and the Boltzmann factor,

$$
q=\sum_{j} d_{j} q_{\mathrm{tr}} q_{\mathrm{rot}} q_{\mathrm{vib}} \exp \left(\frac{-E_{j}}{R T}\right)
$$

The degeneracy and Boltzmann factor account for the contributions from multiple isomers ${ }^{12}$ that are important above the melting temperature of the cluster.

Assuming that the cluster rotates rigidly and vibrates harmonically, the partition function becomes 


$$
\begin{aligned}
q= & \sum_{j} d_{j}\left\{V\left(\frac{2 \pi n m k T}{h^{2}}\right)^{3 / 2}\right\} \\
& \times\left\{\left(\frac{\pi^{1 / 2}}{\sigma_{j}}\right)\left(\frac{8 \pi^{2} k T}{h^{2}}\right)^{3 / 2}\left(I_{A} I_{B} I_{C}\right)^{1 / 2}\right\} \\
& \times\left\{\prod_{i=1}^{3 n-6} \frac{\exp \left(-h \nu_{i} / 2 k T\right)}{1-\exp \left(-h \nu_{i} / k T\right)}\right\} \exp \left(\frac{-E_{j}}{R T}\right),
\end{aligned}
$$

where $m$ is the mass of each particle, $\sigma$ is the rotation symmetry number, $\left\{I_{A}, I_{B}, I_{C}\right\}$ are the principal moments of inertia, $V$ is the volume per cluster, $k$ is the Boltzmann constant, $R$ is the gas constant, and $h$ is Planck's constant. We assume that various isomers differ mainly in their degeneracies, binding energies, and symmetry numbers. This is reasonable since the cluster mass $(\mathrm{nm})$ for each isomer is conserved and the product of the principal moments of inertia $\left(I_{A} I_{B} I_{C}\right)$ is nearly constant for these roughly spherical isomers. This assumption will be bourne out by what follows. We further assume that the vibrational frequency distribution $\left\{\nu_{i}\right\}$ is barely changed by the small number of defects defining the differences between isomers. ${ }^{31}$ Also, Eq. (4) assumes fixed volume, restricting these results to the low pressure limit. For a Lennard-Jones gas, the low pressure limit applies for total pressures much less than the characteristic pressure $\left(D_{e} / R_{e}^{3}\right)$ which is about 300 atm for argon. ${ }^{24}$ The LennardJones energy parameter $\left(D_{e}\right)$ and distance parameter $\left(R_{e}\right)$ are discussed further in Sec. VI.

From Eqs. (2) and (4), the free energy can be written as

$$
\begin{aligned}
F= & E^{\min }+\sum_{i=1}^{3 n-6} \frac{h \nu_{i}}{2}-R T \ln \left\{V\left(\frac{2 \pi n m k T}{h^{2}}\right)^{3 / 2}\right\} \\
& -R T \ln \left\{\pi^{1 / 2}\left(\frac{8 \pi^{2} k T}{h^{2}}\right)^{3 / 2}\left(I_{A} I_{B} I_{C}\right)^{1 / 2}\right\} \\
& -R T \sum_{i=1}^{3 n-6} \ln \left\{\frac{1}{1-\exp \left(-h \nu_{i} / k T\right)}\right\} \\
& -R T \ln \left\{\sum_{j}\left(\frac{d_{j}}{\sigma_{j}}\right) \exp \left(-\frac{E_{j}-E^{\min }}{R T}\right)\right\},
\end{aligned}
$$

where $E^{\text {min }}$ denotes the global minimum potential energy of the cluster.

The last term in Eq. (5), called the configurational free energy, is used to define a configurational partition function,

$$
q_{\text {config }}=\sum_{j}\left(\frac{d_{j}}{\sigma_{j}}\right) \exp \left(-\frac{E_{j}-E^{\min }}{R T}\right) .
$$

This definition differs from previous definitions ${ }^{12}$ because of the inclusion of the symmetry number $\left(\sigma_{j}\right)$. We feel that its inclusion is justified since $\sigma_{j}$ is much more isomerdependent than the remainder of $q_{\text {rot }}$. This inclusion greatly simplifies the accounting for rotationally equivalent isomers and estimating the rotational symmetry of individual isomers. The details appear in the appendix.

Cheng and Berry ${ }^{18}$ (CB) published an analytic model for estimating the configurational partition function in which at- oms of the outer layer of the cluster are "promoted" to the surface as "floaters", to form the various isomers. In CB, the floaters populate equivalent and noninteracting lattice sites on the cluster surface. The degeneracy $d_{j}$ is estimated as $C_{i}^{j}$, the binomial coefficient for $i$ items selected from $j$, where $j$ is the capacity of the shell.

While quite simple, the CB model has several serious deficiencies, several of which were acknowledged by CB. ${ }^{18}$

1. Holes are arbitrarily restricted to the outermost layer of the cluster.

2. Rotationally equivalent configurations are counted incorrectly as distinct isomers.

3. No allowance is made for the reduction in rotational states by the point symmetry of the isomers.

4. The number of accessible sites changes as the shell is populated. $^{14}$

5. The distinct binding sites on the cluster surface do not have the same energies. ${ }^{14}$

6. Intrashell interactions among the promoted atoms (neglected in $\mathrm{CB}$ ) become progressively more important as the shell becomes more densely filled.

Deficiencies 1-3 are satisfied through our method for constructing isomers and estimating degeneracies from fragments (Sec. IV and the appendix), deficiency 4 is addressed using our shellwise lattice search algorithm (SLS) to identify acceptable fragments (Sec. V), and deficiencies 5-6 are handled via our Linear Group Contribution method (LGC) to estimate the binding energy of each isomer (Sec. VI). Together, SLS/LGC generates the data needed to more accurately estimate $q_{\text {config }}$ [Eq. (6)].

\section{THE CORE PLUS SHELL MODEL}

Since the interaction potential is considered as pairwise additive, we will partition the cluster into a central core and an outer shell and then evaluate their degeneracies and energetics separately,

$$
E_{j}=E^{\text {shell }}+E^{\text {core }}+E^{\text {shell/holes }} .
$$

The core may contain holes due to promoted atoms, but we assume that it retains much of the structure of a Mackay icosahedral cluster. This assumption is supported by simulation results. ${ }^{16-19}$ The shell contains the promoted atoms and the atoms that would be left over after constructing the largest Mackay icosahedral cluster with all of the given atoms. In Sec. VI we describe a method for calculating the energy of interactions among shell atoms and with a dense core. We use a mean-field estimate to account for the energetics of holes in the core which is also described in Sec. VI.

The degeneracy associated with Eq. (7) is written as

$d_{j}^{\text {rot }}=d^{\text {shell }} \cdot d^{\text {core }}$,

where the superscript "rot" is a reminder that Eq. (8) overestimates the number of distinct isomers due to rotational equivalence. Rotational equivalence is a special case of permutation of equivalent atoms. No such permutation can produce distinguishable isomers. Two tasks remain: to calculate 
the degeneracy and energy of the core itself, and to avoid overcounting of rotationally equivalent configurations.

The core, in turn, can be viewed as composed of an inner core and a full or nearly-filled outer shell. Thus, the method described in Eqs. (7) and (8) can also be applied to the core of the full cluster. This procedure can be applied recursively until the remaining atoms form a cluster for which the energy is known. (In practice, this is either a complete Mackay icosahedral cluster or a cluster with fewer than 13 atoms.) Mathematically, the procedure is as follows:

$$
\begin{aligned}
& E_{j}-E^{\mathrm{min}}=\Delta E^{\text {holes }}+\sum_{\alpha=1}^{N} \Delta E_{\alpha, c}^{\text {shell }}, \\
& \Delta E_{\alpha, c}^{\text {shell }}=E_{\alpha, c}-E_{\alpha}^{\text {min }}, \\
& \Delta E^{\text {holes }}=\sum_{\alpha=1}^{N-1} \sum_{\beta=\alpha+1}^{N} E_{\alpha, \beta}^{\text {shell/holes }}, \\
& d_{j}^{\text {rot }}=\prod_{\alpha=1}^{N} d_{\alpha, c}^{\text {shell }}
\end{aligned}
$$

where $N$ is the largest shell containing particles, $\alpha$ and $\beta$ are shell indices, and $c$ specifies the configuration of shell $\alpha$. Specifying a set of shell configuration indices $\left\{c_{1}, c_{2}, \ldots, c_{N}\right\}$ implies the number of filled sites $\left\{f_{1}, f_{2}, \ldots, f_{N}\right\}$ and thus the number of holes $\left\{h_{1}, h_{2}, \ldots, h_{N}\right\}$ in each shell. Together, these sets are equivalent to specifying a unique isomer index $(j)$ since the inter-shell interactions are estimated using a mean-field approach (through $\Delta E_{\alpha, c}^{\text {shell }}$ and $\Delta E^{\text {holes}}$ ).

In the appendix we demonstrate that

$$
\frac{d_{j}}{\sigma_{j}}=\frac{d_{j}^{\mathrm{rot}}}{g},
$$

where

$$
g=\left\{\begin{array}{l}
120, \quad \text { for the } 19-\text { atom PIC cluster }, \\
60, \quad \text { otherwise }
\end{array}\right.
$$

Polyicosahedral clusters (PIC) are rare special cases. See the Appendix for further details. The simplicity of Eq. (13) relies on the inclusion of $\sigma_{j}$ in the definition of $q_{\text {config }}$ [Eq. (6)]. Thus, the 2nd and 3rd deficiencies listed in Section III are much easier to address simultaneously than separately.

Equations (6), (9), (12), and (13) provide a systematic form for calculating $q_{\text {config }}$,

$$
\begin{aligned}
q_{\mathrm{config}}= & \frac{1}{g} \sum_{h} \exp \left(\frac{-\Delta E^{\mathrm{holes}}}{R T}\right) \\
& \times\left\{\prod_{\alpha=1}^{N}\left[\sum_{c} d_{\alpha, c}^{\text {shell }} \exp \left(\frac{-\Delta E_{\alpha, c}^{\text {shell }}}{R T}\right)\right]\right\} .
\end{aligned}
$$

In Sec. V we present methods to estimate the degeneracies $\left(d_{\alpha, c}\right)$ of the various shells. In Sec. VI we present methods to estimate the energetics of the shells $\left(\Delta E_{\alpha, c}^{\text {shell }}\right)$ and of the holes $\left(\Delta E^{\text {holes }}\right)$.
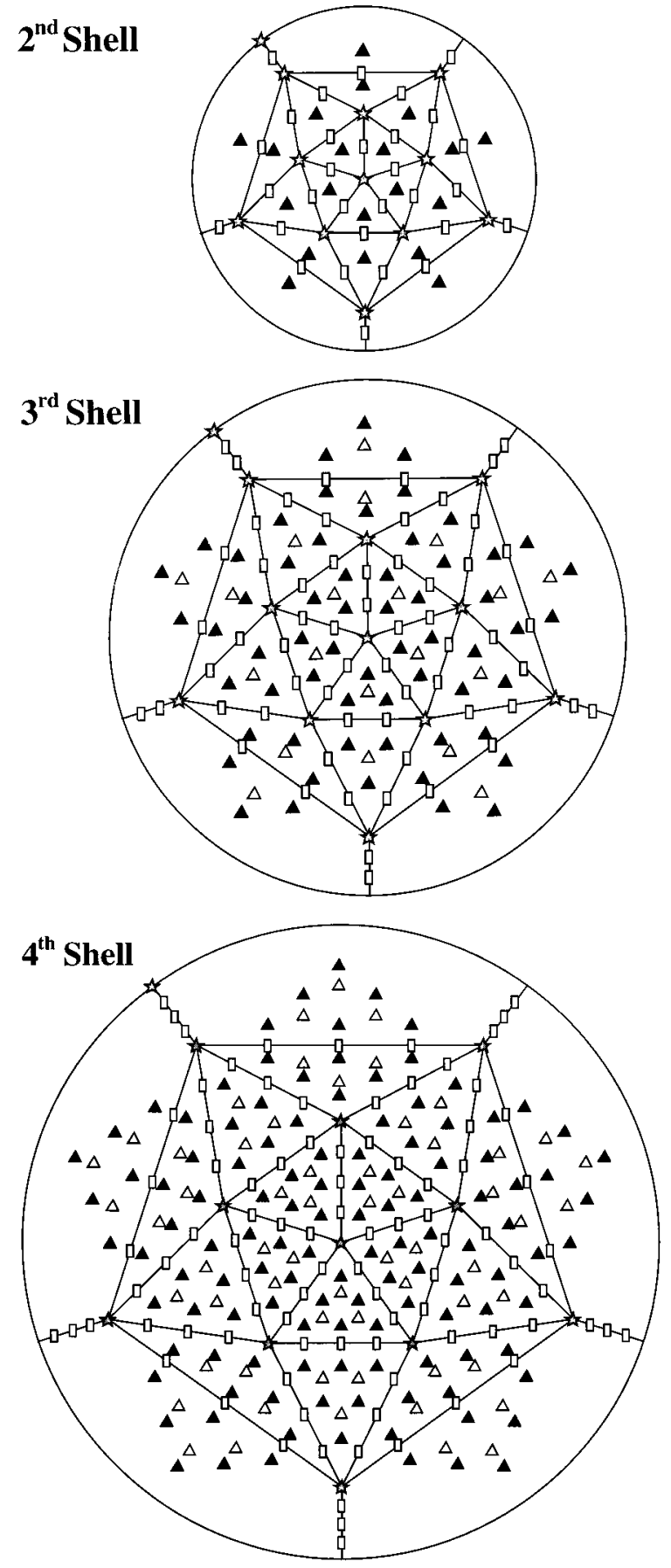

FIG. 2. Projection of the 2nd, 3rd, and 4th shells showing the lattice sites:

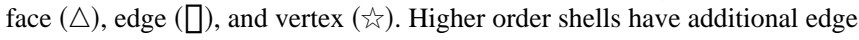
and face sites. White symbols belong to the IC sublattice, black symbols to the FC sublattice, and stars to both lattices.

\section{DEGENERACIES}

\section{A. Lattices and site filling rules (SFR)}

Northby ${ }^{14}$ used a lattice-based method to identify candidates for the minimum potential energy of clusters with partially filled outer shells and dense Mackay icosahedral cores. He used projections of the cluster surface similar to the ones in Fig. 2. Table I categorizes the lattice sites for the first four shells: 
TABLE I. Types of lattice sites for the first four shells. $Z$ is the coordination number with atoms in the underlying shell.

\begin{tabular}{|c|c|c|c|c|c|}
\hline Shell & Type of sites & $Z$ & Sublattice & Quantity & $\begin{array}{l}\text { Shell capacity } \\
\left(f^{\max }\right)\end{array}$ \\
\hline \multirow[t]{2}{*}{0} & Central & $\cdots$ & $\cdots$ & 1 & \\
\hline & & & & & 1 \\
\hline \multirow[t]{2}{*}{1} & Vertex & 1 & IC & 12 & \\
\hline & & & & & 12 \\
\hline \multirow[t]{4}{*}{2} & Faces & 3 & $\mathrm{FC}$ & 20 & \\
\hline & Edge & 2 & IC & 30 & \\
\hline & Vertex & 1 & $\mathrm{IC} / \mathrm{FC}$ & 12 & \\
\hline & & & & & 42 \\
\hline \multirow[t]{5}{*}{3} & Centered face & 3 & IC & 20 & \\
\hline & Off-center face & 3 & $\mathrm{FC}$ & 60 & \\
\hline & Edge & 2 & IC & 60 & \\
\hline & Vertex & 1 & $\mathrm{IC} / \mathrm{FC}$ & 12 & \\
\hline & & & & & 92 \\
\hline \multirow[t]{6}{*}{4} & Type 1 face & 3 & FC & 60 & \\
\hline & Type 2 face & 3 & $\mathrm{FC}$ & 60 & \\
\hline & Type 3 face & 3 & IC & 60 & \\
\hline & Edge & 2 & IC & 90 & \\
\hline & Vertex & 1 & $\mathrm{IC} / \mathrm{FC}$ & 12 & \\
\hline & & & & & 162 \\
\hline
\end{tabular}

1. Face sites with three underlying neighbors

2. Edge sites balanced on two underlying neighbors

3. Vertex sites perched atop one underlying neighbor Northby also defined sublattices which can be completely filled:

IC. The icosahedral sublattice is filled in the Mackay icosahedral structures. IC sites are either shaded white or stars in Fig. 2.

FC. The face-centered sublattice contains those sites which are not part of the IC sublattice and the vertex sites (which are part of both sublattices). In Fig. 2, the FC sites are either shaded black or stars.

From an analysis of the global minimum potential energy structures by Northby, we obtain the following generalized site filling rules (SFR):

SFR 1. FC sites can be filled if the adjacent IC sites are empty.

SFR 2. Edge sites can be filled if the adjacent face sites are empty and at least two of the adjacent IC sites are filled.

SFR 3. Vertex sites can be filled if at least four of the five adjacent edge sites or all five adjacent face sites are filled.

Rule 1 is due to hard core repulsions between neighboring sites. Rules 2 and 3 arise because edge and vertex sites are not at local minima with respect to the core and must therefore be stabilized by other atoms in the shell.

We use the site filling rules (SFRs) to screen proposed isomers in a Monte Carlo sampling algorithm. First, configurations are generated by filling randomly selected lattice sites. Then the rules are applied to determine if the configuration is valid. If it is valid, the configuration is recorded so that its energy can be estimated. (See Sec. VI for the estimation procedure.) The ratio of the number of accepted configurations to the number of trial configurations estimates the
TABLE II. Shell capacity $\left(f^{\max }\right)$, number of lattice sites $(L)$, and sample space size $(S)$ for enumerating all lattice fillings (up to their capacity) for three shells.

\begin{tabular}{cccc}
\hline \hline Shell & 2 & 3 & 4 \\
\hline$f^{\max }$ & 2 & 3 & 4 \\
$L$ & 62 & 152 & 282 \\
$S$ & $\sum_{f=1}^{42} C_{f}^{62}$ & $\sum_{f=1}^{92} C_{f}^{152}$ & $\sum_{f=1}^{162} C_{f}^{282}$ \\
& $\approx 4.6 \times 10^{18}$ & $\sim 5.7 \times 10^{45}$ & $\approx 7.7 \times 10^{84}$ \\
\hline \hline
\end{tabular}

proportion of valid configurations among the configurations in the sample space. Standard binomial statistics are used to place error estimates on that ratio. ${ }^{32}$ In the balance of Sec. V we describe two sample spaces and corresponding sampling procedures (BSA and TBSA) which are applied to the 2nd and 3rd shells.

\section{B. The building shell algorithm (BSA)}

The simplest procedure for identifying acceptable isomers is to start with the empty lattice described above and fill some of the sites. Configurations with more filled sites than the shell capacity need not be considered. Table II gives the size of the sample space for applying this method to the 2nd through the 4th shell. Clearly, there are too many configurations to enumerate them all.

We use a Monte Carlo sampling procedure to estimate the total number of acceptable configurations and to identify particular configurations representative of the isomer distribution. Conceptually, we partition the sample space into sections with each section having the same number of filled sites $(f)$ in shell $\alpha$ and then sample from each section separately. (In practice, all sections were done concurrently, but each section was analyzed separately.) Let $x_{c}$ be the number of configurations of type $c$ out of $X$ randomly selected configurations. Configuration $c$ has $f$ filled lattice sites in a shell having $L$ lattice sites. Then the estimated degeneracy of configuration $c$ is the product of the fraction of acceptable configurations $\left(P_{c}\right)$ and the size of the sample space $\left(S_{f}\right)$,

$$
\begin{aligned}
& d_{c}^{\text {shell }}=P_{c} \cdot S_{f}, \\
& P_{c}=\frac{x_{c}}{X} .
\end{aligned}
$$

The size of the sample space $\left(S_{f}\right)$ is the number of combinations of $L$ lattice sites taken $f$ at a time,

$$
S_{f}=C_{f}^{L}=\frac{L !}{(L-f) ! f !} .
$$

We report a 95\% confidence interval for the composite acceptance ratio $(P)$ based on the Gaussian approximation to the binomial distribution (appropriate if $\min [x, X-x] \geqslant 5$ ), ${ }^{32}$

$$
\begin{aligned}
& x=\left.\sum_{c} x_{c}\right|_{f}, \\
& P=\left.\sum_{c} P_{c}\right|_{f}=\frac{x}{X} \pm 1.96 \sqrt{\frac{x}{X^{2}}\left(1-\frac{x}{X}\right)} .
\end{aligned}
$$


TABLE III. Number of iterations $(X)$ and computation time for implementation of two sampling algorithms applied to two shells.

\begin{tabular}{ccccc}
\hline \hline Shell & Algorithm & $X$ & Computer & Computation time \\
\hline 2 & BSA & $10^{9}$ & IBM RISC 6000 & 3 wks elapsed time \\
2 & TBSA & $1.024 \times 10^{8}$ & Intel Touchtone Delta & $1.5 \mathrm{~h}$ elapsed time \\
& & & & $770 \mathrm{~h}$ cpu time \\
3 & BSA & $10^{9}$ & Intel Touchstone Delta & $4.5 \mathrm{~h}$ elapsed time \\
& & & & $2300 \mathrm{~h} \mathrm{cpu} \mathrm{time}$ \\
3 & TBSA & $2.00192 \times 10^{7}$ & Intel Touchstone Delta & $12 \mathrm{~h} \mathrm{elapsed} \mathrm{time}$ \\
& & & $6200 \mathrm{~h} \mathrm{cpu} \mathrm{time}$ \\
\hline \hline
\end{tabular}

Table III gives the number of trial configurations $X$ and the computation time for investigations of the 2nd and 3rd shells using this building shell algorithm (BSA). An IBM RISC 6000 was used to investigate the 2nd shell. The Intel Touchstone Delta ${ }^{33}$ computer was used for the balance of the sampling runs.

Figure 3 gives the acceptance ratio $(P)$ for the 2 nd and 3rd shells as a function of the number of filled sites $(f)$ using BSA. This figure illustrates the deficiency of BSA. The acceptance ratio is too small $\left(P<10^{-6}\right)$ to get a statistically meaningful sample for all but the most dilute $(f<20)$ sections. BSA is useful, however, as a check on the more practical (but more complicated) algorithm presented below and as a subroutine in that algorithm.

\section{The tearing then building shell algorithm (TBSA)}

Hard core repulsions between neighboring FC and IC sites (SFR 1) are the most common cause for rejecting conformations in the BSA. This suggests using a sampling algorithm which uses these repulsions to reduce the sample size $(S)$ rather than to reject conformations. We describe this tearing then building shell algorithm (TBSA) next.

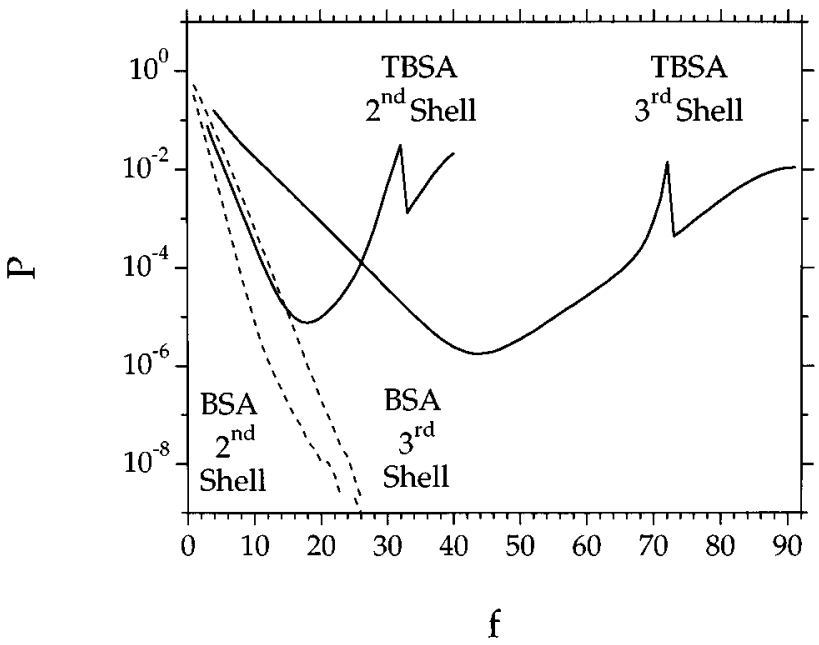

FIG. 3. Acceptance ratio $(P)$ as a function of the number of filled lattice sites $(f)$ for two sampling algorithms (BSA and TBSA) applied to the 2nd and 3rd shells. For TBSA, filling of the FC sublattice leads to a decrease in $(P)$ beyond $f=32$ for the 2 nd shell and $f=72$ for the 3 rd shell.
Whereas BSA began with an empty shell, each of the $X$ iterations of the TBSA begins with a filled IC shell. First, some of the IC atoms are randomly selected for removal from the IC shell. Next, the FC sites are investigated to determine which would be free of hard-core repulsions if they were filled. Subshells with IC filled sites and FC available sites are tabulated in $x(I C, F C)$. Then, $Y$ iterations of the BSA are used to sample the available FC sites. Finally, SFR 2 and 3 are used to screen the composite $(I C \cup F C)$ shell conformations. Acceptable conformations of type $c$ are tabulated in $y_{c}$. As with the BSA, the estimated number of configurations is the product of an acceptance ratio $\left(P_{c}\right)$ and a sample space size $\left(S_{c}\right)$

$$
\begin{aligned}
& d_{c}^{\text {shell }}=P_{c} \cdot S_{c}, \\
& P_{c}=p 1 \pm 1.96 \sqrt{\frac{p 1(1-p 1)}{Y \sum_{i=F C}^{F C^{\max } x(I C, i)}},} \\
& p 1=\frac{y_{c}}{Y \sum_{i=F C}^{F C^{\max }} x(I C, i)} .
\end{aligned}
$$

The major improvement over the BSA is the reduced sample size; however, that advantage comes at the cost of a more complicated analysis. Since the number of available FC sites is not known a priori, TBSA requires that the size of the sample space $\left(S_{c}\right)$ be estimated along with the acceptance ratios $(p 1, p 2)$,

$$
\begin{aligned}
S_{c}= & \frac{\left(I C^{\max }\right) !}{\left(I C^{\max }-I C\right) !(I C) !}\left\{\sum_{i=F C}^{F C^{\max }} p 2\left(\frac{i !}{(i-F C) !(F C) !}\right)\right. \\
& \left. \pm 1.96 \frac{\sqrt{\sum_{i=F C}^{F C^{\max }} p 2(1-p 2)\left(\frac{i !}{(i-F C) !(F C) !}\right)^{2} / X}}{\sum_{i=F C}^{F C^{\max }} p 2\left(\frac{i !}{(i-F C) !(F C) !}\right)}\right\},
\end{aligned}
$$

$p 2=x(I C, i) / X$.

The factor in front of the braces in Eq. (23) is the number of ways of removing IC of the icosahedral atoms in the first stage of the TBSA. The term within the braces represents the number of ways of filling in FC of the available face- 


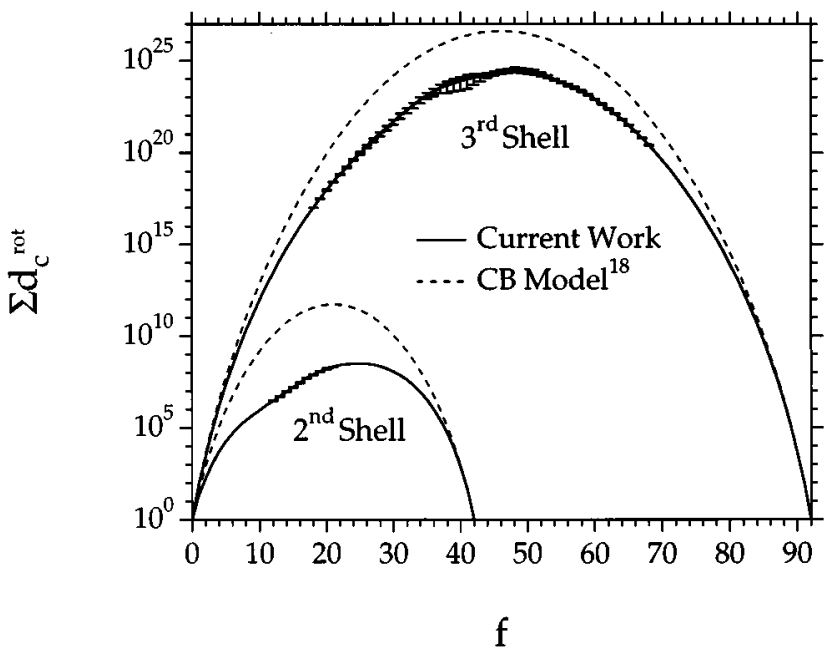

FIG. 4. The number of geometrically distinguishable shell isomers $\left(\sum d_{c}^{\text {rot }}\right)$ (including rotationally equivalent isomers) as a function of the number of filled lattice sites $(f)$. Solid lines represent the results of this study. For clarity, the $95 \%$ confidence limits have been omitted for cases where the error is less than $2 \%$ of the total. Dashed lines (CB) are the estimates of Cheng and Berry (Ref. 18) which do not account for the internal structure of the shells.

centered sites. We used the same approximation ${ }^{32}$ to the binomial distribution as in Eq. (19) and standard rules for error propagation ${ }^{34}$ in deriving the $95 \%$ confidence limits on $P_{c}$ and $S_{c}$. Figure 4 shows that SLS identifies far fewer valid shell configurations than the CB estimate. ${ }^{18}$ This demonstrates that properly treating the internal structure of the shells reduces the number of acceptable configurations, particularly for shells that are roughly half filled.

Table III gives the number of trial configurations $X$ and the computation time on the Intel Touchstone Delta ${ }^{33}$ computer for investigations of the 2nd and 3rd shells using TBSA.

Figure 3 gives the composite acceptance ratio,

$$
P=\left.\sum_{c} p 1 \cdot p 2\right|_{f}=\sum_{c} \frac{y_{c}}{\left.f X Y\right|_{f}},
$$

for the 2nd and 3rd shells as a function of the number of filled sites $(f)$ using TBSA. The worst acceptance ratios are $P=10^{-5}$ for the 2nd shell and $P=10^{-6}$ for the 3rd shell, justifying the added complexity of TBSA.

\section{ENERGETICS}

\section{A. Interaction potential}

To this point, we have not specified an interaction potential between particles. The discussion and results of Sec.V are purely geometric. We have merely assumed that the particles pack like spheres and that their interactions are pairwise additive. Therefore, any two-body radial interaction potential could be used to estimate the energetics of the isomers fragments identified using BSA or TBSA. We use the Lennard-Jones potential to take advantage of previously published minimum energy structures ${ }^{14}$ and correlations for the temperature-dependent contributions to the free energy, ${ }^{35}$

$$
\begin{aligned}
& \epsilon=\rho^{-12}-2 \rho^{-6}, \\
& \epsilon=E / D_{e}, \\
& \rho=r / R_{e} .
\end{aligned}
$$

Some typical Lennard-Jones parameters $\left(R_{e}, D_{e}\right)$ are tabulated $^{36}$ in Table IV.

\section{B. Linear group contribution (LGC) method for estimating the shell energy}

The lattice method described in Section $V$ provides information which can be used to estimate the difference in energy between various configurations. In principle, each of the accepted configurations could be used as a starting point for an off-lattice energy minimization and the resulting energy could be recorded. However, Fig. 4 shows that there are far too many configurations for that approach to be practical. Instead we use a linear group contribution (LGC) method which efficiently estimates shell energies by explicitly including the main interactions, but only indirectly including

\begin{tabular}{|c|c|c|c|c|c|}
\hline Quantity & Units & $\mathrm{Ne}$ & $\mathrm{Ar}$ & $\mathrm{Kr}$ & $\mathrm{Xe}$ \\
\hline$m$ & (amu) & 20.179 & 39.948 & 83.80 & 131.30 \\
\hline$D_{e}$ & $(\mathrm{kcal} / \mathrm{mol})$ & 0.0726 & 0.237 & 0.325 & 0.446 \\
\hline$R_{e}$ & $(\AA)$ & 3.249 & 3.867 & 4.109 & 4.465 \\
\hline$1 \quad \longdiv { D _ { e } }$ & $(\mathrm{GHz})$ & 60.12 & 64.86 & 49.35 & 42.50 \\
\hline$\nu_{\text {char }}=\frac{{ }^{2 \pi R_{e}}}{2} \sqrt{\frac{e}{m}}$ & $\left(\mathrm{~cm}^{-1}\right)$ & 2.004 & 2.162 & 1.645 & 1.417 \\
\hline$E_{\text {char }}=h \nu_{\text {char }}$ & $(\mathrm{cal} / \mathrm{mol})$ & 5.729 & 6.181 & 4.704 & 4.051 \\
\hline$T_{\text {char }}=\frac{h \nu_{\text {char }}}{k}$ & $(\mathrm{~K})$ & 2.883 & 3.111 & 2.367 & 2.039 \\
\hline$I_{\text {char }}=m R_{e}^{2}$ & $\left(\mathrm{amu} \AA^{2}\right)$ & 213.0 & 597.4 & 1415 & 2618 \\
\hline$T_{\text {boil }}$ & $(\mathrm{K})$ & 27.1 & 87.3 & 119.8 & 165.0 \\
\hline$k T_{\text {boil }} / D_{e}$ & $(-)$ & 0.742 & 0.732 & 0.732 & 0.735 \\
\hline
\end{tabular}
more distant pairs,

TABLE IV. Parameters and characteristics quantities for sample systems. ${ }^{36}$ There are two energy scales, $D_{e}$ and $h \nu_{\text {char }} . D_{e}$ determines the potential energy at the optimal structure while $h \nu_{\text {char }}$ determines the zero-point energy and vibrational contributions. Boiling point data is from Ref. 41. 
TABLE V. Linear group contribution (LGC) parameters $\left(E_{i} / D_{e}\right)$ for two potentials. The parameters based on the Lennard-Jones potential are similar to those based on the square-well potential, indicating that the LennardJones-based pareameters are dominated by first-neighbor interactions.

\begin{tabular}{lcccc}
\hline \hline \multirow{2}{*}{$\begin{array}{l}\text { Potential } \\
\text { shell }\end{array}$} & $\begin{array}{c}\text { Square well } \\
\text { (all) }\end{array}$ & 2nd & 3rd & 4th \\
\cline { 3 - 5 } & & & & \\
Intershell interactions & 3 & 3.518 & 4.217 & N/A \\
$\quad$ Centered face & 3 & N/A & 4.213 & 4.612 \\
$\quad$ Off-center face & 2 & 2.184 & 2.690 & \\
$\quad$ Edge & 6 & 5.968 & 6.279 & \\
$\quad$ Vertex supported by FC particles & & 0.588 & 1.195 & \\
$\quad$ Vertex supported by IC particles & 1 & & & \\
Intrashell interactions & 1 & 1.015 & 0.994 & 1.004 \\
$\quad$ FC/FC & 1 & 1.522 & 1.241 & \\
$\quad$ IC/IC & 1 & 1.276 & 1.200 & \\
$\quad$ Edge/vertex & 0 & -0.470 & -0.148 & \\
5-member ring of face particles & 0 & -0.460 & 0.383 & \\
5-member ring of edge particles & & & & \\
\hline \hline
\end{tabular}

${ }^{a}$ This group contribution includes five face/vertex interactions since they are implied by SFR 3. (See Sec. V.)

$$
E_{\mathrm{LGC}}^{\mathrm{shell}}=-\sum_{i} N_{i} E_{i}
$$

In Eq. (29), $N_{i}$ is the number of occurrences of the $i$ th group and $E_{i}$ is the energy contribution of that group. LGC places isomers having similar energies into the same bin. Each bin is defined to contain isomers with the same set $\left\{N_{i}\right\}$. This is efficient since a small number of bins suffice to represent a huge number of total isomers. Each of the group contributions in the model were fitted to differences in energy between previously published minimum potential energy configurations. ${ }^{14}$ By fitting to minimum energy configurations, we expect the energy parameters $\left(E_{i}\right)$ to implicitly include contributions from non-first neighbor pairs that are not explicitly counted in the LGC model. The groups and their energy contributions are given in Table $\mathrm{V}$ for two potentials.

Figure 5 shows that the LGC model reproduces the binding energy of Northby's structures ${ }^{14}$ to within $1 \%$ or $0.5 D_{e}$. The accuracy of this fit justifies the use of the LGC method to estimate the energy of other structures. There are slight systematic trends in the residuals due to the effect of 2 nd and more distant neighbors. Thus, the precision of the shell energy estimates can be improved through the use of the (published) minimum energy configurations,

$$
\Delta E^{\text {shell }} \equiv E^{\text {shell }}-E_{\text {min }}^{\text {shell }} \approx\left(E^{\text {shell }}-E_{\min }^{\text {shell }}\right)_{\text {LGC }} .
$$

For this reason, the LGC method is used to estimate energy differences between clusters with different surface shells. Recall that $E^{\text {shell }}$ is the energy of interaction among the shell atoms and with a dense core. Hole effects are covered below.

Although the LGC parameters are fitted to Eq. (30), they have physical interpretations. The intershell interaction terms reflect the interactions between shell atoms and a dense core. The FC/FC, IC/IC, and edge/vertex terms reflect intrashell interactions. There is no edge/face interaction term since configurations containing such interactions have high energies due to hard-core repulsions. ( $E_{e / f}$ would be very large and positive, which is the basis for SFR 1.) There is no separate face/vertex interaction term since its energetics are confounded by the requirement that a vertex atom be surrounded by all five face atoms. The corresponding edge/ vertex interaction is not confounded since four edge atoms are sufficient to support a vertex atom (SFR 3).

Two subtle effects lead to contributions from fivemembered rings. The first effect involves the loss of one easy

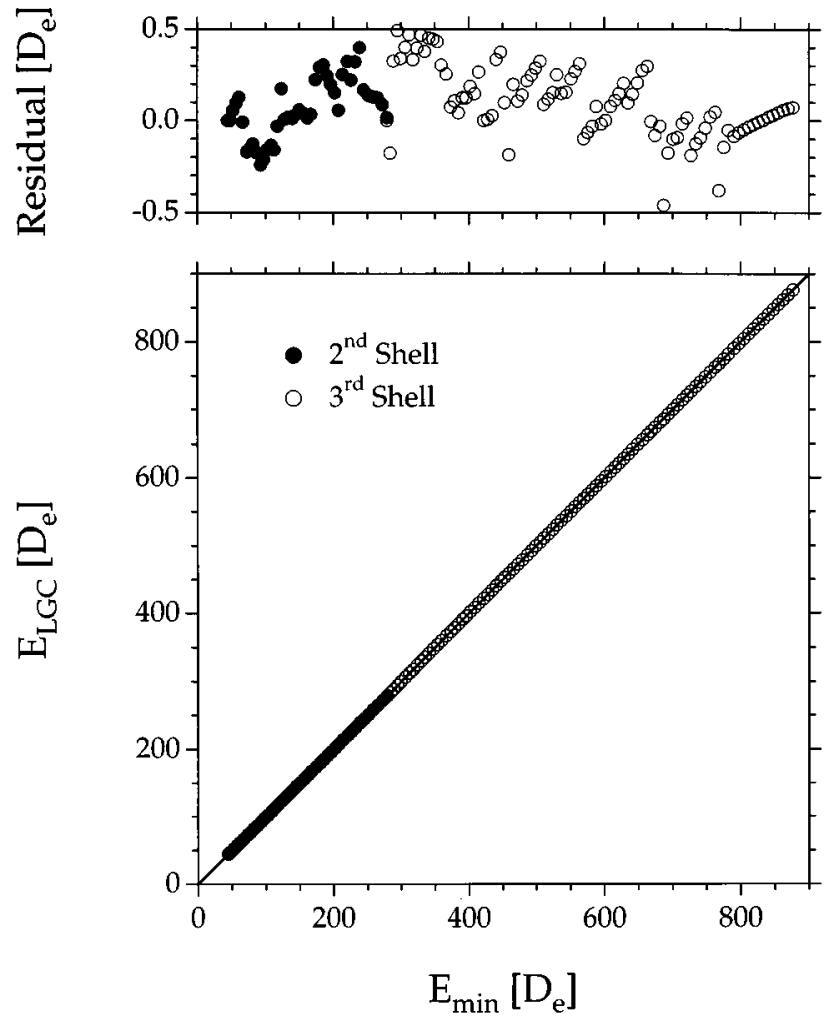

FIG. 5. Cluster energy $\left(E_{\min }\right)$ as determined from off-lattice minimization (Ref. 14) versus the LGC prediction $\left(E_{\mathrm{fit}}\right)$. The line $y=x$ represents perfect agreement. Since the LGC method faithfully reproduces the published results, we use it to estimate binding energies of other structures. The residual $E_{\mathrm{LGC}}-E_{\min }$ is shown separately; it remains smaller than $0.5 D_{e}$. 
TABLE VI. Mean-field estimate of the hole interaction energy for a single hole interacting with a single occupied lattice site, $E_{\alpha, \beta}^{\text {shell/holes }} /\left(D_{e} h_{\alpha} n_{\beta}\right)$. See Eq. (31).

\begin{tabular}{|c|c|c|c|}
\hline $\begin{array}{l}\text { Hole } \\
\text { index } \\
(\alpha)\end{array}$ & $\begin{array}{c}\text { Shell } \\
\text { index } \\
(\beta)\end{array}$ & $\begin{array}{c}\text { Hole interaction } \\
\text { energy } \\
\left(E_{\alpha, \beta}^{\text {shell/holes }} / D_{e} h_{\alpha} n_{\beta}\right)\end{array}$ & $\begin{array}{c}\text { Cluster } \\
\text { sizes } \\
\text { used (Ref. 37) }\end{array}$ \\
\hline 1 & 2 & 0.2212 & 14,55 \\
\hline 1 & 3 & 0.02374 & 56,147 \\
\hline 1 & 4 & 0.002823 & 148,309 \\
\hline 2 & 3 & 0.07861 & 56,147 \\
\hline 2 & 4 & 0.008270 & 148,309 \\
\hline 3 & 4 & 0.04034 & 148,309 \\
\hline
\end{tabular}

degree of freedom for the relaxation of atoms within the shell. For five atoms in a ring, there are five pairs of which any four pairs can relax across the saddles between lattice sites. Simultaneous relaxation of the 5th pair would shorten the circumference of the ring causing all five atoms to "climb" the hard core of the vertex particle's potential. This implies that all five pairs cannot simultaneously reduce the cluster energy by relaxing across a saddle. Therefore, the first effect tends to make the five-membered ring contribution negative. The second effect involves 2 nd neighbors. Since the number of five-membered rings correlates with the number of distant pairs (which are not explicitly included in the LGC method), the five-membered ring contributions include this positive effect. Therefore, the five-membered ring group contributions are a balance between these competing effects.

\section{Hole energy}

We use a mean-field approach to calculate the interaction energy between holes in shell $\alpha$ and the atoms in shell $\beta$ $\left(E_{\alpha, \beta}^{\text {shell/holes }}\right)$. For each of the $h_{\alpha}$ holes in shell $\alpha$, we use the average energy of interaction $(\epsilon)$ between particles at the $L_{\alpha}$ lattice sites in shell $\alpha$ and particles at all of the $L_{\beta}$ lattice sites in shell $\beta$. The distances $\left(r_{t, u}\right)$ between the hole sites $(t)$ and particle sites $(u)$ are estimated using the corresponding distances between particles in minimized dense clusters. ${ }^{37}$

$$
E_{\alpha, \beta}^{\text {shell/holes }}=f_{\beta} h_{\alpha} \sum_{t=1}^{L_{\alpha}} \sum_{u=1}^{L_{\beta}} \frac{\epsilon\left(r_{t, u}\right)}{L_{\alpha} L_{\beta}} .
$$

Conceptually useful subsets of the isomer distribution can be characterized by the total number of holes $(h)$,

$$
h=\sum_{\alpha=1}^{N} h_{\alpha}
$$

and the total number of particles $(n)$,

$$
n=\sum_{\beta=1}^{N} f_{\beta} .
$$

Since the relative orientations of shells $\alpha$ and $\beta$ do not appear in Eq. (31), this is a mean-field estimate. Values for $E_{\alpha, \beta}^{\text {shell/holes }}$ are summarized in Table VI.
TABLE VII. Isomer energies for the 55-atom cluster.

\begin{tabular}{lcccc}
\hline \hline$j$ & Structure & $\begin{array}{c}E_{j}^{39} \\
\left(D_{e}\right)\end{array}$ & $\begin{array}{c}E_{j}^{L G C} \\
\left(D_{e}\right)\end{array}$ & $d_{j} / \sigma_{j}$ \\
\hline 0 & $\begin{array}{c}\text { Mackay Icosahedron } \\
\text { 2nd shell vertex vac., } \\
\text { face-centered cap }\end{array}$ & -279.248 & -279.248 & $1 / 60$ \\
2 & $\begin{array}{c}\text { 2nd shell vertex vac., } \\
\text { off-center cap }\end{array}$ & -276.597 & -276.418 & 4 \\
3 & $\begin{array}{c}\text { 2nd shell edge vac., } \\
\text { face-centered cap }\end{array}$ & -274.090 & -279.482 & 10 \\
4 & $\begin{array}{c}\text { 2nd shell edge vac., } \\
\text { off-center cap }\end{array}$ & & -273.479 & 30 \\
5 & $\begin{array}{c}\text { 1st shell vacancy, } \\
\text { face-centered cap } \\
\text { 1st shell vacancy, } \\
\text { off-center cap }\end{array}$ & & -267.791 & 4 \\
$7+\quad$ Many others ... & & -267.788 & 12 \\
\hline \hline
\end{tabular}

\section{Distribution of inherent structures}

The LGC method along with the mean-field estimate for the hole interaction energy can be used to generate the distribution of cluster inherent structures from the purely geometric data in Section V. We will illustrate using the 55atom cluster because we can compare with other detailed studies. ${ }^{18,24,38,39}$ With zero holes $(h=0)$, there is only one unique minimal structure, with a rotational symmetry number of 60 . With one hole $(h=1)$, the hole can reside at a vertex site in the first shell or at an edge or vertex in the second shell. Furthermore, the promoted atom can occupy one of 20 centered local minima or 60 off-center local minima in the third shell. This gives rise to six additional groups of structures. (See Table VII.) The off-lattice energies for four of these isomers have been calculated explicitly by Doye and Wales. ${ }^{39}$ Their values are reproduced in Table VII and are consistent with the previously stated accuracy of the LGC method. $\left( \pm 0.5 D_{e}\right)$. Allowing additional holes leads to a huge number of inherent structures with higher energies.

Figure 6 shows the convergence of the SLS/LGC method for the 55-atom cluster as a function of the maximum number of holes. To interpret Fig. 6, note that $\left(E-E^{\min } \leqslant n k T_{b} / 2 \approx 21 D_{e}\right)$ corresponds to the normal boiling point. Thus a maximum of six holes is sufficient to generate the classically accessible structures at the normal boiling point. Our results are similar to Ref. 38 (based on simulated quenching) with the differences arising from our inclusion of the rotational symmetry number and from the finite sample generated by quenching. Also shown is the prediction of the $\mathrm{CB}$ model. ${ }^{18} \mathrm{By}$ ignoring the structure of the various shells, this model tends to over-estimate the number of inherent structures, particularly for non-magic-numbered clusters. It should be noted that Cheng and Berry chose to apply their model only to magic number clusters. Subsequent modeling studies should be tested on magic number and nonmagic number clusters to avoid this bias.

Figure 7 shows the cumulative distribution of structures as a function of binding energy for clusters having 15 to 140 


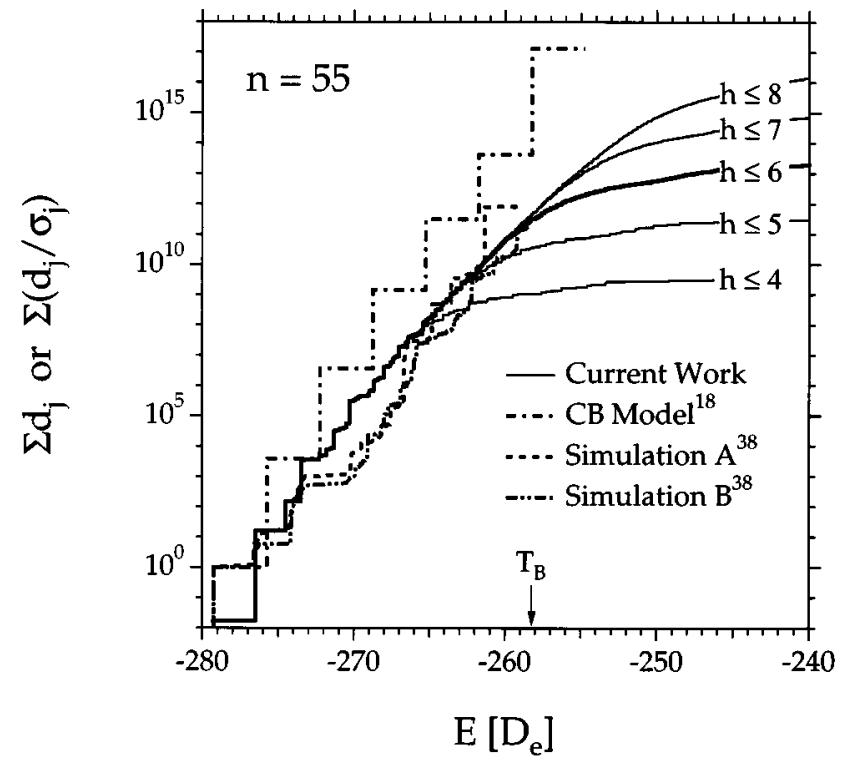

FIG. 6. Cumulative number of isomers $\left(\Sigma_{E_{j} \leqslant E} d_{j}^{\text {rot }}\right)$ as a function of binding energy $(E)$ for a cluster of 55 particles. The curves demonstrate the effect of limiting the total number of holes $(h)$. We find that six holes are sufficient to generate most cluster isomers contributing significantly to the partition function at temperatures up to the normal boiling point $\left(E-E_{\min } \approx 21 D_{e}\right.$ or $E=-258 D_{e}$ ). The prediction of the Cheng and Berry model (Ref. 18) and results based on simulations by Doye and Wales (Ref. 38) are also shown for comparison.

atoms. (For clarity, only every fifth cluster size is shown.) Up to six holes $(h \leqslant 6)$ were used in generating these structures. Although a huge number of isomers are observed, the total falls far short of a simple extrapolation of the Tsai and Jordan results for $n \leqslant 13 .^{25}$ As pointed out by Doye and Wales ${ }^{38}$ this observation suggests that there are additional isomers at higher energies but that these structures are not thermally accessible at reasonable temperatures.

In Sec. V, we argued that there are too many shell configurations to enumerate them all; however, it is conceivable that, in some cases, all of the bins of configurations were identified. This is possible since configurations with similar energies were binned together. Our sampling was sufficient to contain the minimum energy structures as reported by Northby ${ }^{14}$ for the following ranges: $13 \leqslant n \leqslant 30,41 \leqslant n \leqslant 63$, and $140 \leqslant n \leqslant 147$. This suggests that most of the structures in these size ranges have been identified. In the intermediate ranges, there are simply too many structures to expect an undirected search to find most of the structures in the time available. Still, sampling is sufficient to represent the distribution of structures. Since only a small fraction of low energy structures seem to contribute to the configurational partition function, improved estimates for $q_{\text {config }}$ could be obtained using the LGC method and an acceptance sampling Monte Carlo algorithm.

\section{DISCUSSION AND APPLICATIONS}

\section{A. Extrapolation to bulk properties}

In a previous paper, ${ }^{35}$ we showed that a quadratic fit to a plot of free energy per particle $(F / n)$ versus $n^{-1 / 3}$ yields estimates for the bulk chemical potential $\left(\mu_{0}\right)$, bulk surface tension $\left(\sigma_{0}\right)$, and Tolman length $(\delta)$,

$$
\frac{F}{n}=\mu+\sigma_{0}(4 \pi)^{1 / 3}(3 v)^{2 / 3} n^{-1 / 3}-2 \sigma_{0} \delta(4 \pi)^{2 / 3}(3 v)^{1 / 3} n^{-2 / 3} \text {. }
$$

The Tolman length gives the first correction to the surface tension $(\sigma)$ due to the radius of curvature $(r)$,

$$
\sigma(r)=\sigma_{0}\left(1-\frac{2 \delta}{r}\right)+O\left(r^{-2}\right) .
$$

The molecular volume $(v)$ is assumed to be constant to be consistent with the assumed form for the partition function (4).

We calculated cluster free energies $(F)$ using Eq. (5) and the following estimates (from a previous paper in this $\left.\operatorname{series}^{35}\right)$ for the binding energy $\left(E^{\mathrm{min}}\right)$ :

$$
\begin{aligned}
E^{\min } /\left(n D_{e}\right)= & -8.610+15.744 n^{-1 / 3}-11.894 n^{-2 / 3} \\
& +17.194 n^{-1}-20.387 n^{-4 / 3}
\end{aligned}
$$

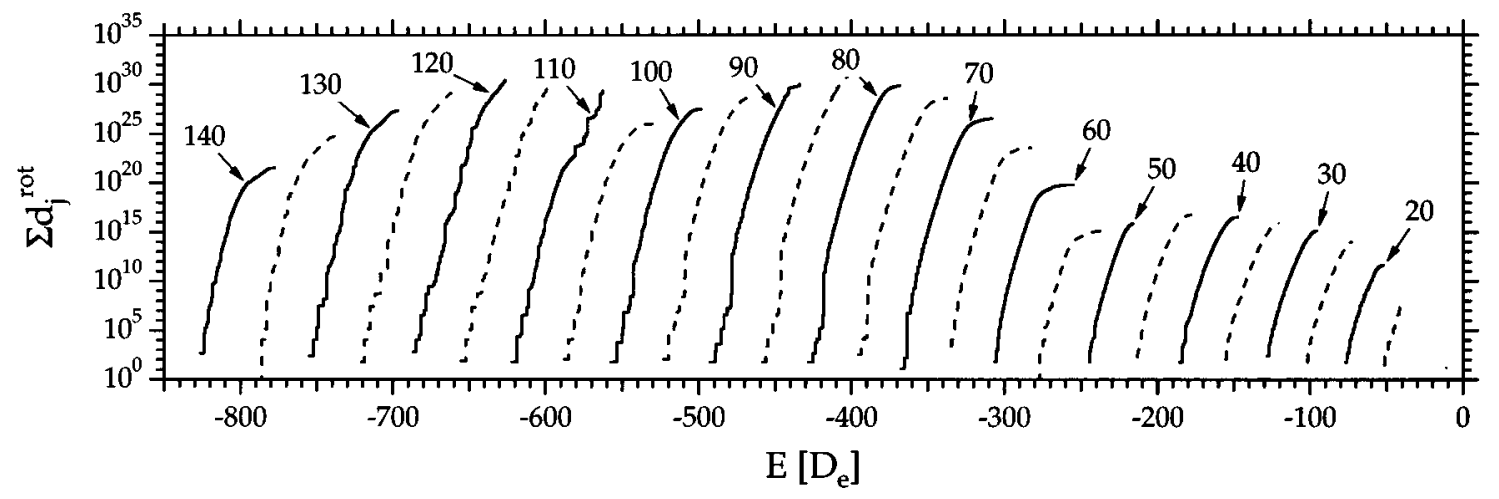

FIG. 7. Cumulative number of isomers $\left(\Sigma_{E_{j} \leqslant E} d_{j}^{\text {rot }}\right)$ as a function of binding energy $(E)$ and number of particles $(n)$. The summation was carried out for up to 6 holes $(h \leqslant 6)$. 


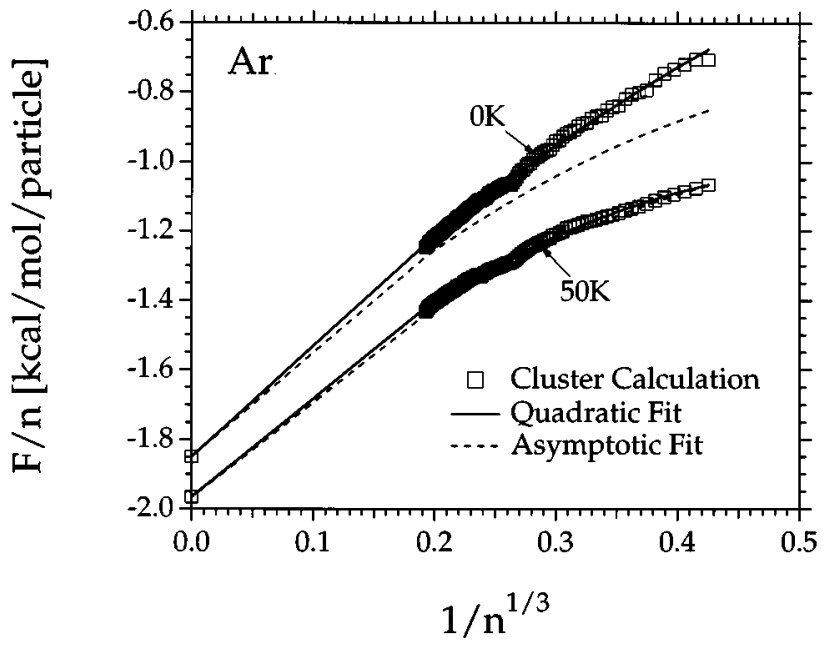

FIG. 8. Calculated free energy per particle vs $n^{-1 / 3}$ for argon as motivated by Eq. (34). The intercept is the bulk chemical potential, the slope at the origin is proportional to the surface tension, and the curvature is related to the Tolman length.

the zero point energy $\left(E^{z p}\right)$,

$$
\begin{aligned}
& E^{z p}=\frac{h}{2} \sum_{i}^{3 n-6} \nu_{i}, \\
& E^{z p} \approx(3 n-6) h \nu_{\text {char }}\left[10.287-\frac{7.679}{N}+\frac{5.422}{N^{2}}-\frac{1.439}{N^{3}}\right],
\end{aligned}
$$

the product of the principal moments of inertia $\left(I_{A} I_{B} I_{C}\right)$,

$$
\begin{aligned}
\left(I_{A} I_{B} I_{C}\right)^{1 / 3} \approx & I_{\text {char }} \sum_{y=1}^{N} y^{2}\left(10 y^{2}+2\right)\left[0.4298+\frac{0.0104}{N}\right. \\
& \left.+\frac{0.2457}{N^{2}}-\frac{0.0666}{N^{3}}\right],
\end{aligned}
$$

and the vibrational free energy $\left(F_{\text {vib }}\right)$,

$$
\begin{aligned}
& F^{\mathrm{vib}}=R T \sum_{i=1}^{3 n-6} \ln \left[1-\exp \left(-\frac{h \nu_{i}}{k T}\right)\right], \\
& F^{\mathrm{vib}} \approx \xi R T(3 n-6) \ln \left[1-\exp \left(-\frac{\theta_{E}}{T}\right)\right], \\
& \xi=\exp \left(\frac{\alpha\left(\theta_{E}-\theta_{\min }\right)}{T(T+\alpha)}\right), \\
& \frac{\alpha}{T_{\text {char }}}=1.05+\frac{1.97}{N}-\frac{1.88}{N^{2}}+\frac{0.47}{N^{3}}, \\
& \frac{\theta_{E}}{T_{\text {char }}}=19.27-\frac{16.95}{N}+\frac{15.42}{N^{2}}-\frac{5.40}{N^{3}}, \\
& \frac{\theta_{\text {min }}}{T_{\text {char }}}=0+\frac{17.16}{N}-\frac{14.49}{N^{2}}+\frac{4.67}{N^{3}} .
\end{aligned}
$$

\begin{tabular}{|c|c|c|c|c|}
\hline Solid & $\begin{array}{c}T \\
(\mathrm{~K})\end{array}$ & $\begin{array}{c}\mu \\
\left(\frac{\mathrm{kcal} / \mathrm{mol}}{\text { particle }}\right)\end{array}$ & $\begin{array}{c}\sigma \\
\left(\frac{\mathrm{dyn}}{\mathrm{cm}}\right)\end{array}$ & $\begin{array}{c}\delta \\
(\AA)\end{array}$ \\
\hline \multirow[t]{2}{*}{$\mathrm{Ne}$} & 0 & -0.448 & 17.2 & 0.74 \\
\hline & 10 & -0.449 & 17.0 & 0.74 \\
\hline \multirow[t]{6}{*}{$\mathrm{Ar}$} & 0 & -1.852 & 45.2 & 0.81 \\
\hline & 10 & -1.853 & 45.1 & 0.81 \\
\hline & 20 & -1.861 & 44.5 & 0.82 \\
\hline & 30 & -1.884 & 43.8 & 0.83 \\
\hline & 40 & -1.920 & 42.8 & 0.84 \\
\hline & 50 & -1.967 & 41.7 & 0.85 \\
\hline \multirow[t]{8}{*}{$\mathrm{Kr}$} & 0 & -2.653 & 56.3 & 0.85 \\
\hline & 10 & -2.655 & 56.1 & 0.85 \\
\hline & 20 & -2.670 & 55.5 & 0.85 \\
\hline & 30 & -2.703 & 54.7 & 0.86 \\
\hline & 40 & -2.751 & 53.9 & 0.87 \\
\hline & 50 & -2.813 & 52.9 & 0.89 \\
\hline & 60 & -2.886 & 51.9 & 0.90 \\
\hline & 70 & -2.967 & 50.8 & 0.90 \\
\hline \multirow[t]{11}{*}{$\mathrm{Xe}$} & 0 & -3.695 & 66.3 & 0.92 \\
\hline & 10 & -3.697 & 66.1 & 0.92 \\
\hline & 20 & -3.717 & 65.5 & 0.92 \\
\hline & 30 & -3.756 & 64.8 & 0.93 \\
\hline & 40 & -3.813 & 64.1 & 0.94 \\
\hline & 50 & -3.882 & 63.3 & 0.94 \\
\hline & 60 & -3.962 & 62.5 & 0.95 \\
\hline & 70 & -4.054 & 61.7 & 0.96 \\
\hline & 80 & -4.152 & 60.8 & 0.97 \\
\hline & 90 & -4.258 & 59.8 & 0.98 \\
\hline & 100 & -4.371 & 58.7 & 0.99 \\
\hline
\end{tabular}

Planck's constant ( $h$ ) in Eqs. (37), (38), and (40) should not be confused with the number of holes in a cluster. The bind-
TABLE VIII. The bulk chemical potential $(\mu)$, surface tension $(\sigma)$, and Tolman length $(\delta)$ for the solid form of four noble gases, estimated using Eq. (34) and asymptotic analysis on Eqs. (36)-(45). For the $0 \mathrm{~K}$ evaluations, the temperature-dependent contributions to the free energy were set to zero.

ing energy estimate Eq. (36) is based on minimum energy structures for large clusters. ${ }^{40} \mathrm{We}$ use it to estimate bulk properties. For smaller clusters, $(n \leqslant 147)$ tabulated values were used. ${ }^{14}$ The characteristic values $\nu_{\text {char }}, I_{\text {char }}$, and $T_{\text {char }}$ are defined and tabulated for four noble gases in Table IV. Although Eqs. (38), (39), and (43)-(45) were based on magic-numbered clusters, ${ }^{35}$ we have used Eq. (38) and (43)(45) as interpolating functions by inverting (1) to give values for $N$ for clusters containing arbitrary numbers of particles (n) and have used a linear interpolation in Eq. (39).

Figure 8 shows polynomial fits to the free energy of argon clusters at several temperatures as motivated by Eq. (34). Also shown are the asymptotic polynomials which were used to determine bulk properties. Note that translation and rotation contribute terms of order $\ln (n) / n$, which are negligible in the large $n$ limit. Table VIII summarizes the estimated bulk properties of four noble gases and Table IX gives polynomial fits to the cluster free energies for use by the reader. (Tables of cluster free energy as a function of temperature and cluster size are too extensive to publish.)

Since the bulk properties listed in Table VIII were not used to determine the parameters in the atomic potential, ${ }^{36}$ they can be used to check the potential. The chemical potential relative to the ideal gas at $0 \mathrm{~K}$ and zero pressure is related to the vapor pressure as follows: ${ }^{30}$ 
TABLE IX. Free energy per particle based on quadratic fits as in Fig. 8. This data is provided as a concise summary of the cluster free energies which are used in Sec. VII, but are too exhaustive to reproduce.

\begin{tabular}{|c|c|c|}
\hline Solid & $\begin{array}{c}T \\
(\mathrm{~K})\end{array}$ & $\begin{array}{c}F / n \\
\left(\frac{\mathrm{kcal} / \mathrm{mol}}{\text { particle }}\right)\end{array}$ \\
\hline \multirow[t]{2}{*}{$\mathrm{Ne}$} & 0 & $-0.448+0.951 n^{-1 / 3}-0.546 n^{-2 / 3}$ \\
\hline & 10 & $-0.449+0.961 n^{-1 / 3}-0.751 n^{-2 / 3}$ \\
\hline \multirow[t]{6}{*}{$\mathrm{Ar}$} & 0 & $-1.850+3.529 n^{-1 / 3}-1.804 n^{-2 / 3}$ \\
\hline & 10 & $-1.850+3.544 n^{-1 / 3}-2.037 n^{-2 / 3}$ \\
\hline & 20 & $-1.859+3.534 n^{-1 / 3}-2.295 n^{-2 / 3}$ \\
\hline & 30 & $-1.882+3.503 n^{-1 / 3}-2.548 n^{-2 / 3}$ \\
\hline & 40 & $-1.917+3.461 n^{-1 / 3}-2.800 n^{-2 / 3}$ \\
\hline & 50 & $-1.965+3.412 n^{-1 / 3}-3.053 n^{-2 / 3}$ \\
\hline \multirow[t]{8}{*}{$\mathrm{Kr}$} & 0 & $-2.653+4.967 n^{-1 / 3}-2.480 n^{-2 / 3}$ \\
\hline & 10 & $-2.655+4.982 n^{-1 / 3}-2.735 n^{-2 / 3}$ \\
\hline & 20 & $-2.670+4.961 n^{-1 / 3}-3.000 n^{-2 / 3}$ \\
\hline & 30 & $-2.703+4.922 n^{-1 / 3}-3.254 n^{-2 / 3}$ \\
\hline & 40 & $-2.751+4.874 n^{-1 / 3}-3.502 n^{-2 / 3}$ \\
\hline & 50 & $-2.813+4.824 n^{-1 / 3}-3.753 n^{-2 / 3}$ \\
\hline & 60 & $-2.885+4.770 n^{-1 / 3}-4.005 n^{-2 / 3}$ \\
\hline & 70 & $-2.967+4.712 n^{-1 / 3}-4.255 n^{-2 / 3}$ \\
\hline \multirow[t]{11}{*}{$\mathrm{Xe}$} & 0 & $-3.715+6.898 n^{-1 / 3}-3.408 n^{-2 / 3}$ \\
\hline & 10 & $-3.717+6.911 n^{-1 / 3}-3.677 n^{-2 / 3}$ \\
\hline & 20 & $-3.737+6.886 n^{-1 / 3}-3.948 n^{-2 / 3}$ \\
\hline & 30 & $-3.776+6.844 n^{-1 / 3}-4.206 n^{-2 / 3}$ \\
\hline & 40 & $-3.833+6.794 n^{-1 / 3}-4.458 n^{-2 / 3}$ \\
\hline & 50 & $-3.902+6.742 n^{-1 / 3}-4.707 n^{-2 / 3}$ \\
\hline & 60 & $-3.983+6.688 n^{-1 / 3}-4.954 n^{-2 / 3}$ \\
\hline & 70 & $-4.074+6.632 n^{-1 / 3}-5.200 n^{-2 / 3}$ \\
\hline & 80 & $-4.172+6.575 n^{-1 / 3}-5.450 n^{-2 / 3}$ \\
\hline & 90 & $-4.278+6.516 n^{-1 / 3}-5.698 n^{-2 / 3}$ \\
\hline & 100 & $-4.391+6.455 n^{-1 / 3}-5.950 n^{-2 / 3}$ \\
\hline
\end{tabular}

$$
P^{\mathrm{vap}}=\frac{(2 \pi m)^{3 / 2}(k T)^{5 / 2}}{h^{3}} \exp \left(\frac{\mu}{k T}\right) .
$$

We compare vapor pressures calculated using the estimated bulk chemical potential (Table VIII) with experimental data $^{41,42}$ in Fig. 9. The agreement is excellent up to about $60 \%$ of the normal boiling point. We overestimate the vapor pressure at the boiling point by about $30 \%$, probably due to the harmonic approximation that tends to underestimate the entropy of the solid at elevated temperatures.

Figures 10 and 11 compare the calculated surface tension and Tolman length with the available data. ${ }^{43-45}$ Based on these comparisons with experimental data, we are confident in applying SLS/LGC to noble gas clusters up to $60 \%$ of the normal boiling point.

\section{B. Homogeneous nucleation rates}

As a further application of SLS/LGC, we will use the thermodynamic data in Table VII and the cluster free energies calculated using Eq. (5) to predict the flux of nuclei $J$ from a super-saturated vapor. According to classical theory, ${ }^{46,47}$ the flux $\left(J_{n}\right)$ of clusters through a size $(n)$ is

$$
J_{n}=\alpha_{n} \beta A_{n} C_{n}-E_{n+1} C_{n+1},
$$

where $\alpha_{n}$ is the accommodation coefficient (commonly assumed to be unity), $\beta$ is the flux of monomers through a unit

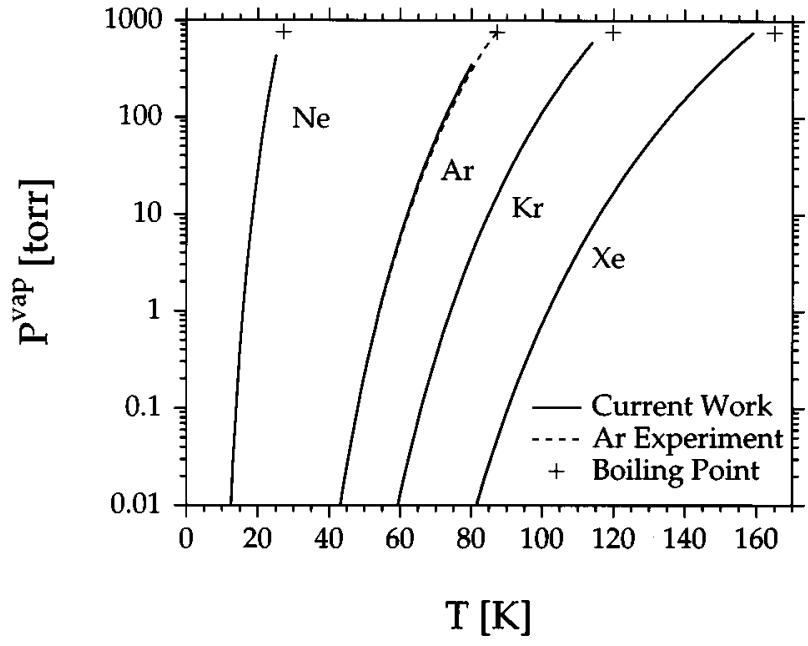

FIG. 9. Calculated vapor pressure for four noble gases. Also shown are the normal boiling points ${ }^{41}$ and the experimental vapor pressure for argon. ${ }^{42}$ The slight deviations near the boiling points are the result of anharmonicity of the vibrational modes.

area, $A_{n}$ is the surface area of the cluster, $C_{n}$ is the number concentration, and $E_{n}$ is the frequency of monomer evaporation from an $n$-mer. To determine $E_{n}$, we follow the approach of Katz. ${ }^{47}$ Applying detailed balancing at full thermodynamic equilibrium yields

$$
J_{n}^{\mathrm{eq}}=0=\alpha_{n} \beta^{\mathrm{eq}} A_{n} C_{n}^{\mathrm{eq}}-E_{n+1}^{\mathrm{eq}} C_{n+1}^{\mathrm{eq}} .
$$

Assuming that the evaporation rate is independent of the vapor conditions leads to the following estimate for $E_{n+1}$ :

$$
E_{n+1}=\alpha_{n} \beta^{\mathrm{eq}} A_{n} C_{n}^{\mathrm{eq}} / C_{n+1}^{\mathrm{eq}} .
$$

Substituting Eq. (49) into Eq. (47),

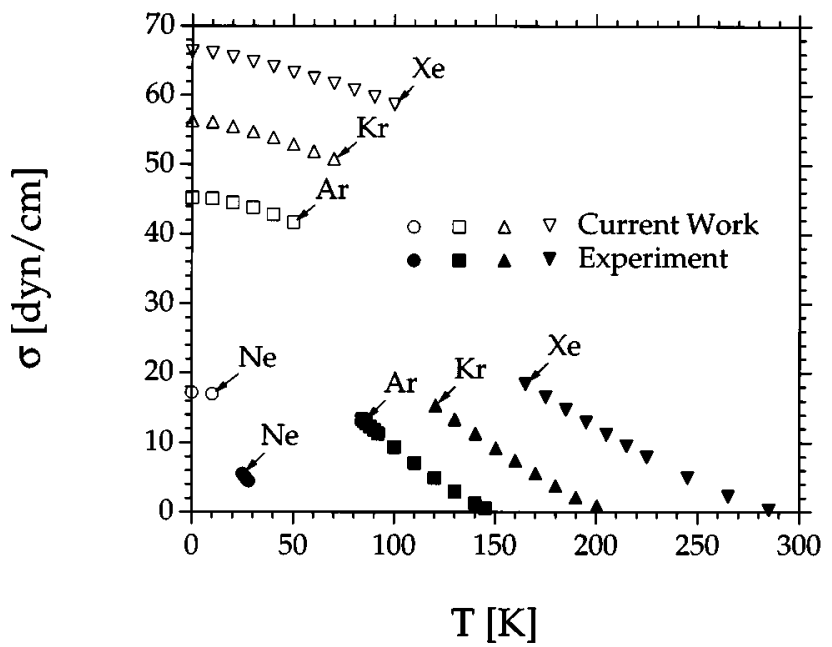

FIG. 10. Calculated and experimental (Refs. 43,44) surface tension for four noble gases. 


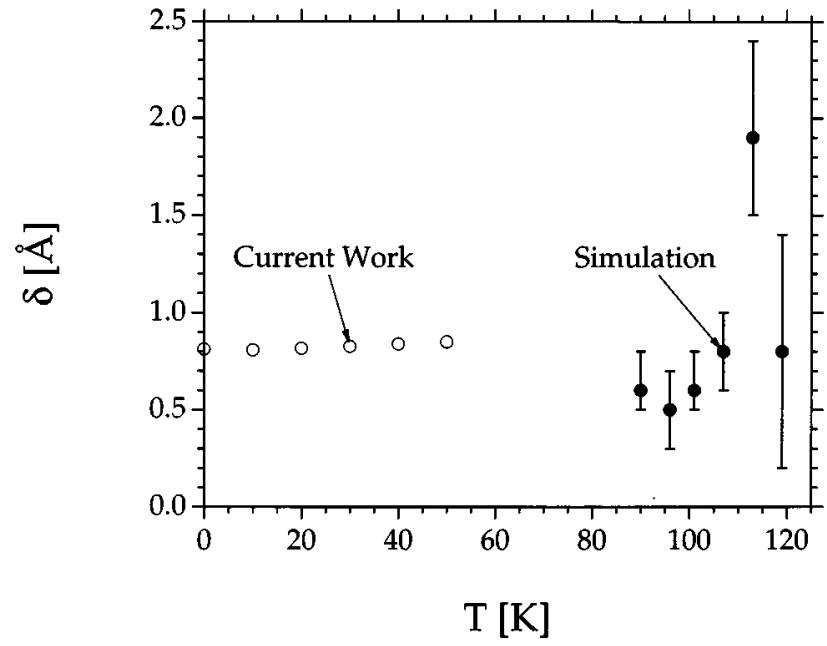

FIG. 11. Calculated and simulated (Ref. 45) Tolman length for argon. The error bars on the simulation results are $\pm 1 \sigma$.

$$
J_{n}=\alpha_{n} \beta A_{n} C_{n}^{\mathrm{eq}}\left[\frac{C_{n}}{C_{n}^{\mathrm{eq}}}-\frac{\beta^{\mathrm{eq}} C_{n+1}}{\beta C_{n+1}^{\mathrm{eq}}}\right],
$$

multiplying and dividing by $\left(\beta^{\mathrm{eq}} / \beta\right)^{n}$, and rearranging gives

$$
\frac{J_{n}}{\alpha_{n} \beta A_{n} C_{n}^{\mathrm{eq}}\left(\beta / \beta^{\mathrm{eq}}\right)^{n}}=\frac{C_{n}}{C_{n}^{\mathrm{eq}}}\left(\frac{\beta^{\mathrm{eq}}}{\beta}\right)^{n}-\frac{C_{n+1}}{C_{n+1}^{\mathrm{eq}}}\left(\frac{\beta^{\mathrm{eq}}}{\beta}\right)^{n+1} .
$$

For steady state nucleation, $J_{1}=J_{2}=\cdots=J_{n}=J$. Summing Eq. (51) over $n$ yields

$J \sum_{n=1}^{b} \frac{1}{\alpha_{n} \beta A_{n} C_{n}^{\mathrm{eq}}\left(\beta / \beta^{\mathrm{eq}}\right)^{n}}=\frac{C_{1}}{C_{1}^{\mathrm{eq}}}\left(\frac{\beta^{\mathrm{eq}}}{\beta}\right)-\frac{C_{b+1}}{C_{b+1}^{\mathrm{eq}}}\left(\frac{\beta^{\mathrm{eq}}}{\beta}\right)^{b+1}$.

From the kinetic theory of gases,

$$
\begin{aligned}
& \beta=P /(2 \pi m k T)^{1 / 2}, \\
& \beta / \beta^{\mathrm{eq}}=P / P^{\mathrm{vap}}=C_{1} / C_{1}^{\mathrm{eq}}=S,
\end{aligned}
$$

where $P$ is the monomer pressure and $S$ is the saturation ratio. By the law of mass action,

$$
\begin{aligned}
& C_{n}^{\mathrm{eq}}=C_{1}^{\mathrm{eq}} \exp \left(-\Delta G_{n} / k T\right), \\
& \Delta G_{n}=F_{n}-n \mu_{\text {bulk }}
\end{aligned}
$$

Finally, using Eqs. (54) and (55) and allowing $b$ to become large gives the desired form for the nucleation rate,

$J=\beta / \sum_{n=1}^{\infty}\left[\alpha_{n} A_{n} C_{1}^{\mathrm{eq}} \exp \left(n \ln S-\frac{\Delta G_{n}}{k T}\right)\right]^{-1}$.

We use the molar volume $(v)$ to estimate the cluster surface area,

$$
A_{n}=(4 \pi)^{1 / 3}(3 n v)^{2 / 3} .
$$

When evaluating $\Delta G_{n}$, it is common to assume that the bulk surface tension applies to the cluster. This is the capillarity approximation,

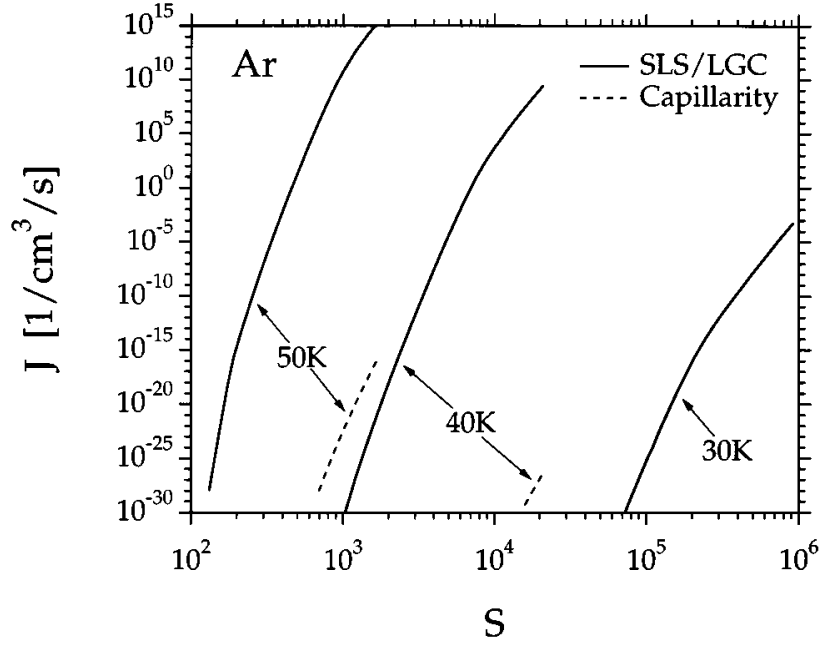

FIG. 12. Nucleation rate $(J)$ as a function of saturation ratio $\left(S=P / P^{\text {vap }}\right)$ for argon. The dotted lines are based on the capillarity approximation using the surface tension extrapolated from the clusters calculations. Therefore, this is an internally consistent test of the capillarity approximation. We find that the capillarity approximation leads to an underestimate of the nucleation rate by $O\left(10^{30}\right)$ at $50 \mathrm{~K}$ and by $O\left(10^{35}\right)$ at $40 \mathrm{~K}$.

$$
\Delta G_{n}^{\mathrm{cap}}=\sigma_{0} A_{n},
$$

Previous workers ${ }^{48,49}$ have compared nucleation rates for argon based on cluster calculations with rates based on the capillarity approximation. Hoare, Pal, and Wegener ${ }^{48}$ performed calculations using only the minimum energy structure for each cluster which limits the results to the purely solidlike (low temperature) limit. Garcia and Torroja ${ }^{49}$ used a purely classical calculation scheme which neglects zeropoint energy contributions to the cluster energy. In both studies, it was unclear whether the comparison tested the capillarity approximation, the model potential used for the cluster calculations, or the simplifying assumptions employed.

We use the extrapolations discussed above to make an internally consistent test of the effect of the capillarity approximation since both our cluster calculations and our estimated bulk properties are based on the same model potential. Furthermore, we verified the model potential using vapor pressure, surface tension, and Tolman length predictions. (See Sec. VII A.) The most limiting assumption used in our calculation of the free energy (5) is the harmonic approximation for the vibrational modes. As stated earlier, this approximation is justified up to about $0.6 T_{b}$. Therefore, we are able to make definitive tests of the capillarity approximation.

Figure 12 shows that the nucleation rate of argon as estimated using cluster calculations is 30 orders of magnitude faster than the capillarity estimate. The discrepancy decreases somewhat with increased temperature.

Despite the discontinuities in the free energy as a function of cluster size (Fig. 8), the nucleation rate is a smooth function of the saturation ratio $(S)$. (See Fig. 12.) This is 


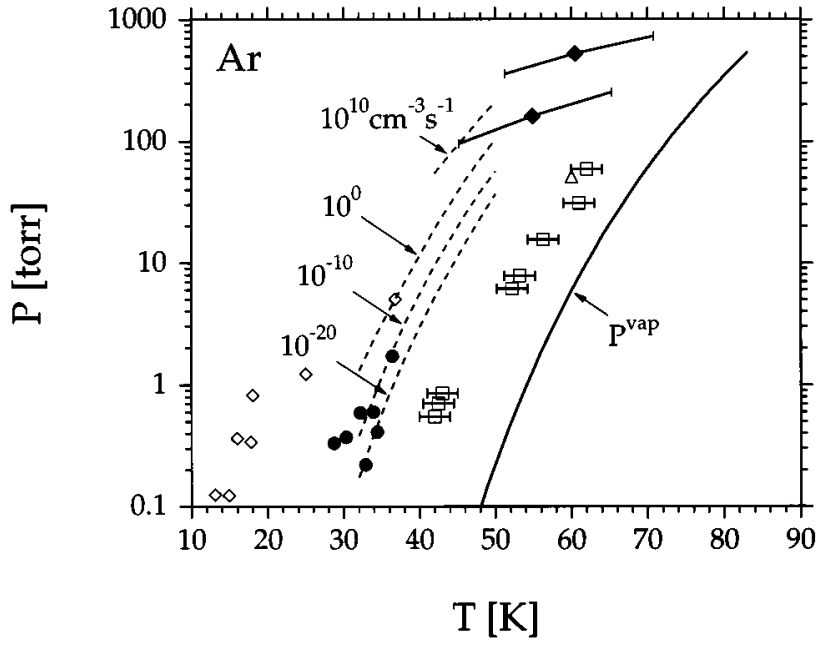

FIG. 13. Nucleation rate $(J)$ as a function of temperature and pressure for argon. The lines are loci of constant nucleation rate (events $/ \mathrm{cm}^{3} / \mathrm{s}$ ) based on the current work. The symbols are experimental determinations of the onset of nucleation (Refs. 50-54) as determined by $(\diamond)$ Pierce et al. (1971), ( $\diamond)$

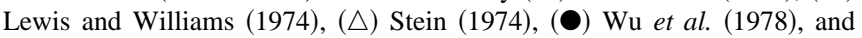
( $\square$ ) Steinwandel and Buchholz (1984).

because the nucleation rate is determined by a summation of terms along a path through cluster space. Although the individual terms are not smooth with respect to their neighbors, each varies smoothly with $S$. Therefore, the path sum (nucleation rate) is a smooth function of $S$ even though the path is a discontinuous function of cluster size. Since the details of the cluster free energy function are not reflected in the nucleation rate, subsequent modeling studies will be able to concentrate on a few cluster sizes and interpolate the data. This will be a huge savings in effort compared to the exhaustive work presented here.

Figures 13 and 14 depict loci of constant nucleation rate as a function of temperature and partial pressure for four noble gases. These curves are bounded due to the limit of applicability of the model $\left(T \leqslant 0.6 T_{b}\right)$ and the range of cluster sizes studied. In order to avoid truncation errors, we only report nucleation rates based on (57) if the summation is dominated by clusters of 17 to 135 atoms. The equilibrium vapor pressure curve calculated using Eq. (46) is also shown in Figs. 13 and 14. The nucleation rate vanishes at the equilibrium curve.

Previous calculations for $\operatorname{argon}^{48,49}$ yielded somewhat higher rates. Most of the discrepancy is due to the use of a formula that is larger than Eq. (57) by a factor of $S$. It is now commonly accepted that Eq. (57) is the correct form. ${ }^{46}$

The available data for argon nucleation, reproduced in Figs. 13, is based on onset determinations. ${ }^{50-54}$ Several researchers determined a locus of points that mark the detection limit of nucleation for their apparatus. A given researcher's detection limit should be relatively insensitive to operating conditions, so each data set in Fig. 13 should follow one of our curves of constant nucleation rate. Since none of them report estimates of their detection limit, only qualitative comparisons are possible. The data by Pierce et al. ${ }^{50}$
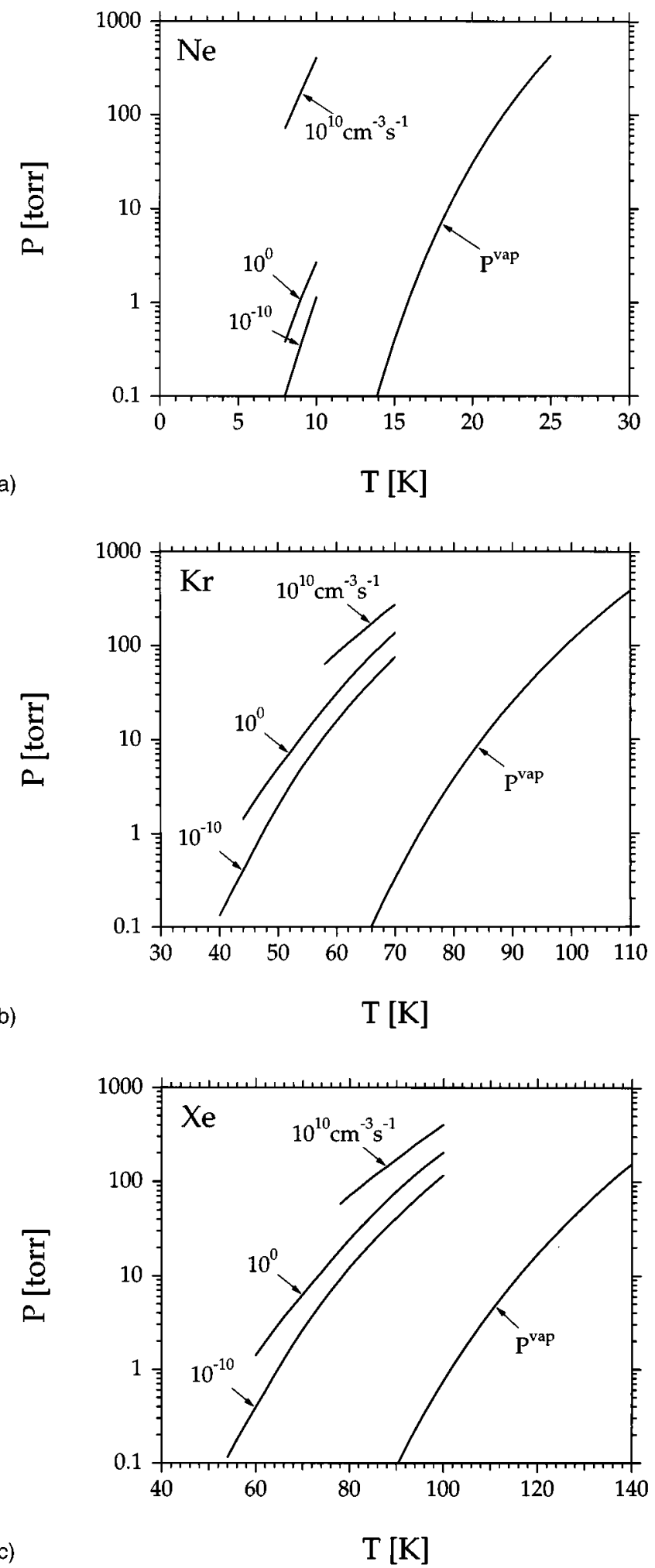

FIG. 14. Nucleation rate $(J)$ as a function of temperature and pressure for three noble gases as in Fig. 13.

and $\mathrm{Wu}$ et $a l . .^{53}$ are generally consistent with our calculations. Error bars on Pierce's data are the author's. Wu's data center on our curve for $10^{-10} \mathrm{~cm}^{-3} \mathrm{~s}^{-1}$ with a scatter of \pm 3 $\mathrm{K}$, suggesting the experimental uncertainty in those trials. Calculated nucleation rates in this temperature range dif- 
fer by 10 orders of magnitude. Realistic detection limits for nucleation in jets are $O\left(10^{10} \mathrm{~cm}^{-3} \mathrm{~s}\right)$. The data by Stein ${ }^{52}$ and by Steinwandel and Buchholz ${ }^{54}$ is too near the saturation curve to be consistent with the current results. (This would imply a sensitivity of roughly $10^{-40} \mathrm{~cm}^{-3} \mathrm{~s}^{-1}$.) The error bars on the latter data set are estimated from Fig. 5 in Ref. 54. Lewis and William's data ${ }^{51}$ imply enormous saturation ratios which seem unlikely in light of this work.

Detailed comparisons with experiments await quantitative nucleation rate measurements for noble gas vapors. Previous experiments have used argon because it is inexpensive and available in very high purity. However, argon has the disadvantage of requiring extremely low temperatures to initiate nucleation. Figures 14(a) and 14(b) show that neon and krypton also require extreme conditions. Future experiments may benefit from the more accessible range of pressures and temperatures appropriate for xenon as depicted in Fig. 14(c). We predict reasonable nucleation rates for xenon vapor at a few tens of Torr at liquid nitrogen temperature $(77 \mathrm{~K})$. At these conditions, it should be possible to use a fixed volume apparatus rather than the continuous flow methods used previously for argon. (See Ref. 55 for brief descriptions of various nucleation apparatus.) Using a fixed volume would offset the higher cost of xenon. Quantitative nucleation rate measurements for xenon would be invaluable in guiding theoretical work in nucleation theory.

\section{ACKNOWLEDGMENTS}

We thank Jon Doye and Professor D. J. Wales for providing the data from their prior work (Ref. 38) which we have included in Figure 5. RBM acknowledges a National Science Foundation Graduate Research Fellowship. Partial support of the work was provided by grants from the NSF (CHE 94-13930, CTS 91-13191, and ASC 92-17368) and from the International Fine Particle Research Institute. Portions of this research were performed using the Intel Delta System operated by Caltech on behalf of the Concurrent $\mathrm{Su}$ percomputing Consortium. We thank Donald Dabdub for his expertise in preparing our code for the Delta System and for handling the scheduling of the runs. The facilities of the MSC are also supported by grants from DOE-BCTR, AlliedSignal Corp., Asahi Chemical, Asahi Glass, Chevron Petroleum Technology Co., Chevron Research Technology Co., Hughes Research Laboratories, and Beckman Institute.

\section{APPENDIX: ROTATIONAL EQUIVALENCE}

As noted above, Eq. (12) overestimates the number of distinct isomers due to the inclusion of rotationally equivalent configurations. Similarly, the number of distinguishable rotational states $\left(q_{\text {rot }}\right)$ should be reduced to account for the rotational symmetry of the isomers. Both issues can be addressed simultaneously with the aid of group theory.

We follow the notation of Wigner. ${ }^{56}$ Script capital letters represent groups of symmetry operations and lowercase letters are integers. Let $\mathscr{C}$ be the group of proper rotations of a dense icosahedral core and let $\mathscr{B}$ be the group of proper rotations of a shell built around that core. By definition, ${ }^{41}$ the symmetry numbers of the dense core and shell $\left(\sigma_{c}\right.$ and $\left.\sigma_{s}\right)$ are the orders of $\mathscr{A}$ and $\mathscr{A}$, respectively.

\section{Case 1: Dense core, coincident centers of symmetry}

For a dense core, the group of proper rotations is $I$ and $\sigma_{c}=60$. The center of symmetry of the core coincides with the atom at the center of the core. In the vast majority of clusters, this atom is unique and must therefore lie at the center of symmetry of the full cluster. (The exceptions are addressed below.) For coincident centers of symmetry, it can be shown ${ }^{56}$ that $\mathscr{B}$ and its $(l-1)$ distinct cosets each have the same order $\left(\sigma_{s}\right)$ and together span the $\sigma_{c}$ elements of $\mathscr{A}$ without repeated elements. Simple accounting of elements leads to

$$
\sigma_{c}=\sigma_{s} l .
$$

Once an orientation for the shell is chosen, the $(l-1)$ rotationally equivalent configurations can be identified in a one-to-one manner with the $(l-1)$ distinct cosets. Therefore, $l$ can be replaced by $d^{\text {rot }}$,

$$
\frac{1}{\sigma_{s}}=\frac{d^{\text {rot }}}{60} \text {. }
$$

The degeneracies $\left(d^{\mathrm{rot}}\right)$ calculated in Sec. IV are for one or more isomers of similar energies. Due to the distributive property of division over addition, binning of isomers does not change the result of Eq. (A2),

$$
\begin{aligned}
& \frac{1}{60} \sum_{j} d_{j}^{\mathrm{rot}} \exp \left(\frac{-\Delta E_{j}}{R T}\right)=\sum_{j}\left(\frac{d_{j}}{\sigma_{j}}\right) \exp \left(\frac{-\Delta E_{j}}{R T}\right), \\
& \frac{d_{j}}{\sigma_{j}}=\frac{d_{j}^{\mathrm{rot}}}{60} .
\end{aligned}
$$

\section{Case 2: Dense core, noncoincident centers of symmetry}

In polyicosahedral (PIC) clusters, ${ }^{57}$ the structure consists of two or more interpenetrating icosahedra. For PIC clusters with an even number of icosahedra, the center of symmetry does not necessarily coincide with the center of symmetry of any of the icosahedra considered separately. Therefore, for a few very special structures, there is no unambiguous choice for the core of the cluster. Then, the analysis of Case 1 does not hold because $\mathscr{A}$ and $\mathscr{B}$ are not groups of symmetry operations with respect to the same center.

An example will help illustrate the situation. The 19atom PIC structure, which has $D_{5 h}$ symmetry, consists of two interpenetrating icosahedra. The center of one icosahedron is a vertex of the other (and vice versa) and they share five other vertex atoms. The center of symmetry of the cluster is midway between the centers of symmetry of the icosahedra considered separately. Therefore, neither of the icosahedra can be unambiguously labeled as the core. For this structure, Eq. (A2) underestimates $\sigma_{s}$ by a factor of two. 
Fortunately, the 19-atom PIC structure is the only structure which has both an ambiguous core assignment and a favorable binding energy. The other PIC structures presented by Farges et al. ${ }^{57}$ have centers of symmetry which are coincident with the center of symmetry of one of its constituent icosahedra. One can construct other PIC structures with ambiguous core assignments, but none of them have large enough binding energies to contribute significantly to $q_{\text {config }}$ due to the Boltzmann weighting in Eq. (6). Therefore, we consider the 19-atom PIC structure as a special case and neglect the error in the estimates of the symmetry numbers of other PIC structures with ambiguous core assignments.

\section{Case 3: Cores containing holes}

For clusters containing holes, two (or more) shells lack some of the symmetry of the core before the promoted atoms left the core. The same argument used in Case 1 can be used here if $\mathscr{b}$ is defined as the group of proper rotations of the core before the promoted atoms left the core and $\mathscr{B}$ is defined as the group of proper rotations of the composite shell composed of all incomplete shells. Therefore, Eq. (A2) also applies for clusters containing holes.

${ }^{1}$ D. Kashchiev, J. Chem. Phys. 76, 5098 (1982); D. W. Oxtoby and D. Kashchiev, ibid. 100, 7665 (1994).

${ }^{2} \mathrm{H}$. Haberland, in Clusters of Atoms and Molecules, edited by $\mathrm{H}$. Haberland (Springer-Verlag, Berlin, 1994), pp. 208-223.

${ }^{3}$ J. Farges, M. F. DeFeraudy, B. Raoult, and G. Torchet, J. Phys. Colloq. 38, C2-47 (1977); J. Chem. Phys. 78, 5067 (1983); 84, 3491 (1986).

${ }^{4}$ S. S. Kim and G. D. Stein, J. Colloid. Interface Sci. 87, 180 (1982).

${ }^{5}$ J. W. Lee and G. D. Stein, Surf. Sci. 156, 112 (1985).

${ }^{6}$ B. Raoult, J. Farges, M. F. DeFeraudy, and G. Torchet, Philos. Mag. B. 60, 881 (1989)

${ }^{7}$ U. Buck in Ref. 2, pp. 232-239.

${ }^{8}$ H. Haberland, Surf. Sci. 156, 305 (1985).

${ }^{9} \mathrm{C}$. Brechignac, Ph. Cahuzac, J. Leygnier, R. Pflaum, and J. Weiner, Phys. Rev. Lett. 61, 314 (1988)

${ }^{10}$ U. Buck and H. Meyer, J. Chem. Phys. 84, 4854 (1986).

${ }^{11}$ U. Buck, Ber. Bunsenges. Phys. Chem. 96, 1294 (1992).

${ }^{12}$ M. R. Hoare, Adv. Chem. Phys. 40, 49 (1979), and references therein.

${ }^{13}$ J. Farges, M. F. DeFeraudy, B. Raoult, and G. Torchet, Adv. Chem. Phys. 70B, 45 (1988), and references therein.

${ }^{14}$ J. A. Northby, J. Chem. Phys. 87, 6166 (1987).

${ }^{15}$ J. A. Northby, J. Xie, D. L. Freeman, and J. D. Doll, Z. Phys. D 12, 69 (1989); J. Xie, J. A. Northby, D. L. Freeman, and J. D. Doll, J. Chem. Phys. 91, 612 (1989).

${ }^{16}$ J. D. Honeycutt and H. C. Andersen, J. Phys. Chem. 91, 4950 (1987).

${ }^{17}$ J. Jellinek, T. L. Beck, and R. S. Berry, J. Chem. Phys. 84, 2783 (1986); T. L. Beck, J. Jellinek, and R. S. Berry, ibid. 87, 545 (1987); T. L. Beck and R. S. Berry, ibid. 88, 3910 (1988); R. S. Berry, Z. Phys. D 12, 161 (1989).

${ }^{18}$ H. P. Cheng and R. S. Berry, Phys. Rev. A 45, 7969 (1992).

${ }^{19}$ H. L. Davis, J. Jellinek, and R. S. Berry, J. Chem. Phys. 86, 6456 (1987).

${ }^{20}$ R. D. Etters and R. Danilowicz, J. Chem. Phys. 71, 4767 (1979).

${ }^{21}$ V. V. Nauchitel and A. J. Pertsin, Mol. Phys. 40, 1341 (1980).

${ }^{22}$ N. Quirke and P. Sheng, Chem. Phys. Lett. 110, 63 (1984).

${ }^{23}$ D. L. Freeman and J. D. Doll, Adv. Chem. Phys. 70B, 139 (1988).
${ }^{24}$ H. P. Cheng, X. Li, R. L. Whetten, and R. S. Berry, Phys. Rev. A 46, 791 (1992).

${ }^{25}$ C. J. Tsai and K. D. Jordan, J. Phys. Chem. 97, 11227 (1993).

${ }^{26}$ R. S. Berry, J. Chem. Soc. Faraday Trans. 86, 2343 (1990).

${ }^{27}$ A. L. Mackay, Acta Cryst. 15, 916 (1962).

${ }^{28}$ R. F. K. Herzog, W. P. Poschenrieder, and F. G. Satkiewicz, Radiat. Effects 18, 199 (1973).

${ }^{29}$ F. H. Stillinger and T. A. Weber, Phys. Rev. A 28, 2408 (1983).

${ }^{30}$ T. L. Hill, Statistical Thermodynamics (Dover, New York, 1986), pp. 104, $161-167$.

${ }^{31}$ F. H. Stillinger and T. A. Weber, J. Chem. Phys. 81, 5095 (1984).

${ }^{32}$ G. J. Hahn and W. Q. Meeker, Statistical Intervals (Wiley, New York, 1991), pp. 104-107.

${ }^{33}$ The Touchstone Delta System is a massively parallel computer with 520 Intel i860 computational processors operated by Caltech on behalf of the Concurrent Supercomputing Consortium.

${ }^{34}$ S. J. Kline and F. A. McClintock, Mech. Eng. 75, 3 (1953).

${ }^{35}$ R. B. McClurg, R. C. Flagan, and W. A. Goddard, III, J. Chem. Phys. 102, 3322 (1995)

${ }^{36}$ The Lennard-Jones parameters used in this study were chosen to agree with the bulk lattice spacing [J. Donohue, Structures of the Elements (Krieger, Malabar, FL, 1982)] and the heat of vaporization at $0 \mathrm{~K}$ after correcting for zero point energy [R. Hultgren, P. D. Desai, D. T. Hawkins, M. Gleiser, K. K. Kelley, and D. D. Wagman, Selected Values of the Thermodynamic Properties of the Elements (American Society for Metals, Metals Park, OH, 1973)]. The method used to calculate Lennard-Jones parameters from this information is detailed elsewhere [N. Karasawa and W. A. Goddard III, J. Phys. Chem. 93, 7320 (1989)].

${ }^{37}$ The coordinates of the atoms in minimized structures were determined using POLY-GRAPH from Molecular Simulations, Inc., Burlington, MA.

${ }^{38}$ J. P. K. Doye and D. J. Wales, J. Chem. Phys. 102, 9659 (1995).

${ }^{39}$ J. P. K. Doye and D. J. Wales, J. Chem. Phys. 102, 9673 (1995).

${ }^{40}$ K-T. Lm, S. Brunett, M. Iotov, R. B. McClurg, N. Vaidehi, S. Dasgupta, S. Taylor, and W. A. Goddard, J. Comp. Chem. (in press).

${ }^{41}$ P. Atkins, Physical Chemistry, 3rd ed. (Freeman, New York, 1986), pp. 531,813 .

${ }^{42}$ W. T. Ziegler, J. C. Mullins, and B. S. Kirk, Technical Report \#2, Project \#A-460, Contract \#CST-7238, National Bureau of Standards, 1962.

${ }^{43}$ K. C. Nadler, J. A. Zollweg, W. B. Streett, and I. A. McLure, J. Colloid. Interface Sci. 122, 530 (1988).

${ }^{44}$ G. A. Cook, Argon, Helium, and the Rare Gases (Interscience, New York, 1961).

${ }^{45}$ M. J. Haye and C. Bruin, J. Chem. Phys. 100, 556 (1994).

${ }^{46}$ D. W. Oxtoby, J. Phys. Condensed Matter. 4, 7627 (1992).

${ }^{47}$ J. L. Katz, Pure Appl. Chem. 64, 1661 (1992).

${ }^{48}$ M. R. Hoare, P. Pal, and P. P. Wegener, J. Colloid. Interface Sci. 75, 126 (1980).

${ }^{49}$ N. G. Garcia and J. M. S. Torroja, Phys. Rev. Lett. 47, 186 (1981).

${ }^{50}$ T. Pierce, P. M. Sherman, and D. D. McBride, Astron. Acta 16, 1 (1971).

${ }^{51}$ J. W. L. Lewis and W. D. Williams, "Argon condensation in free jet expansions,"' Report No. AEDC-TR-74-32, 1974; W. D. Williams and J. W. L. Lewis, Report No. AEDC-TR-76-67, 1976.

${ }^{52}$ G. D. Stein, "Argon condensation in a supersonic nozzle" Report to ONR, available from Technical Information Service No. AD-A007357/ 7GI, 1974.

${ }^{53}$ B. J. C. Wu, P. P. Wegener, and G. D. Stein, J. Chem. Phys. 69, 1776 (1978).

${ }^{54}$ J. Steinwandel and T. Buchholz, Aerosol Sci. Technol. 3, 71 (1984).

${ }^{55}$ R. H. Heist and H. He, J. Phys. Chem. Ref. Data 23, 781 (1994).

${ }^{56}$ E. Wigner, Group Theory (Academic Press, New York, 1959), pp. 58-61.

${ }^{57}$ J. Farges, M. F. DeFeraudy, B. Raoult, and G. Torchet, Surf. Sci. 156, 370 (1985) 